\title{
Gobierno Regional de Arequipa 2005-2018: Ejecución presupuestal y de proyectos de desarrollo y gestión de los sectores educación y salud. Un caso de gestión de gobierno subnacional en el Perú
}

\author{
Regional Government of Arequipa 2005-2018: Budget and \\ development project execution and management of the education and health \\ sectors. A case of subnational government management in Peru
}

Manuel Bedregal Salas

Universidad Católica San Pablo, Arequipa, Perú • mbedregal@ucsp.edu.pe

\section{Resumen}

En este trabajo se evalúa la ejecución presupuestal, la administración de los proyectos de desarrollo y los avances en los sectores Educación y Salud del Gobierno Regional de Arequipa, Perú, entre los años 2005 y 2018. Se encuentra que la gestión presupuestal y la administración de proyectos de desarrollo han sido ineficaces, y lo conseguido en los sectores sociales insuficiente, a pesar del mayor financiamiento asignado. Puede afirmarse que esta instancia de gobierno ha sido deficiente en su contribución con el logro de los objetivos de desarrollo regional.

Palabras clave: descentralización, gobierno regional, Arequipa, ejecución presupuestal, proyectos de desarrollo, sectores sociales, desarrollo económico.

\begin{abstract}
This paper evaluates the budget execution, the administration of development projects and the progress in the Education and Health sectors of the Regional Government of Arequipa, Peru, between 2005 and 2018. It is found that budget management and administration of development projects have been ineffective and what has been achieved in the social sectors insufficient despite the increased funding allocated. It can be affirmed that this level of government has been deficient in its contribution to the achievement of regional development objectives.
\end{abstract}

illustro • Universidad Católica San Pablo, Arequipa • Vol. 12, 2021, 42-78 • e-ISSN 2710-2440

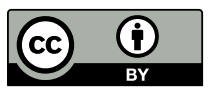


Keywords: decentralization, regional government, Arequipa, budget execution, development projects, social industries, economic development.

\section{Introducción}

Arequipa es una región ubicada en la zona sur occidental del Perú que alberga a 1,382,730 habitantes (INEI, 2108), de los cuales nueve de cada diez viven en zonas urbanas. Para su gobierno administrativo se divide en ocho provincias y 109 distritos, con ciudades ubicadas desde el nivel del mar hasta los 4,467 m s. n. m., en un área de 63,345.39 km2. La provincia de Arequipa alberga al $75 \%$ de la población regional y es un claro polo de desarrollo por la presencia de oportunidades laborales y provisión de servicios básicos. $\mathrm{Al}$ año 2018, la región mantenía bolsones de pobreza $(8.6 \%)$ y extrema pobreza $(1.1 \%)$ (INEI-Enaho, 2018), especialmente en las zonas rurales, con especial incidencia en la población femenina. Arequipa es una de las regiones más desarrolladas del país, según la mayoría de indicadores, y es la quinta que más recursos recibe de las 25 incluidas en el Presupuesto General de la República.

La creación de gobiernos regionales en el Perú marcó un hito en el proceso de descentralización. Si bien la Constitución Política del año 1993 la define como «una forma de organización democrática que constituye una política permanente de Estado, de carácter obligatorio, que tiene como objetivo fundamental el desarrollo integral del país», no fue sino nueve años después que se promulgó la Ley de Bases de la Descentralización, Ley N. ${ }^{\circ} 27783$, que, entre otros objetivos, señala: «Objetivos a nivel económico: a) Desarrollo económico autosostenido y de la competitividad de las diferentes regiones y localidades del país, en base a su vocación y especialización productiva... b) Cobertura y abastecimiento de servicios sociales básicos en todo el territorio nacional; c) Disposición de la infraestructura económica y social necesaria para promover la Inversión en las diferentes circunscripciones del país... d) Redistribución equitativa de los recursos del Estado... e) Potenciación del financiamiento regional y local».

En el marco de esta política de Estado, en el año 2002 se promulgó la Ley Orgánica de Gobiernos Regionales, Ley N. ${ }^{\circ} 27867$, que crea los Gobiernos Regionales, según la cual «los Gobiernos Regionales [...] tienen por finalidad fomentar el desarrollo regional integral sostenible, promoviendo la inversión pública y privada y garantizar el ejercicio pleno de los derechos y la igualdad de oportunidades de sus habitantes». Es pertinente señalar que la regionalización peruana no incluye la autonomía del uso de los recursos recaudados por cada región, sino que se sustenta en transferencias a través de la Ley General de Presupuesto.

Existen documentos valiosos de diagnóstico general del proceso de regionalización en el país (CGR, 2012; Congreso, 2013; Molina, 2016; PCM, 2018), que coinciden en detectar la falta de un norte claro para el modelo adoptado y en la necesidad de relanzarlo para darle un sentido realmente de gestión del desarrollo. En ellos se habla de experiencias fallidas que podrían servir para el aprendizaje y de un avance en el proceso de democratización del país y de la participación ciudadana en la gestión de sus regiones. 
Por otro lado, se señala la falta de claridad sobre los beneficios específicos para la población y sobre la delimitación de competencias con el poder ejecutivo central. También se reconoce la presencia de graves casos de corrupción que desprestigiaron el proceso, así como el cargo de gobernador en sí mismo, afectando la confianza de la población en el proceso de descentralización en su conjunto.

En dichos análisis, se señala que el Perú cuenta con herramientas para hacer seguimiento a la gestión pública que permitirían transparentar la información y crear mecanismos de rendición de cuentas sobre los resultados en la provisión de bienes públicos, pero que estas herramientas no son utilizadas (Molina, 2016).

Los documentos más serios que analizan el proceso concluyen que este no ha sido medido en cuanto a sus resultados ni generado canales para rendir cuentas a la ciudadanía, lo que impide la formación de ideas claras sobre el desempeño de las autoridades regionales, la calidad de su gestión y el grado de cumplimiento de sus ofertas electorales. Coinciden, también, en la necesidad de revisarlo y plantear reformas profundas que signifiquen delimitar funciones y deslindarlas respecto de las del gobierno central. Pese a este consenso, no se ha tomado acciones ni presentado iniciativas legislativas en este sentido.

El proceso de regionalización en Arequipa ha tenido cinco períodos de gestión, cuatro ya concluidos: el gobierno de Daniel Vera, 20032006; los de Juan Manuel Guillén, 2007-2010 y 2011-2014; y el de Yamila Osorio, 2015-2018. Se ha considerado, en la presente investigación, las gestiones culminadas que abarcan un horizonte de 16 años, de 2003 a 2018.
El presente trabajo incluye siete secciones. Luego de esta introducción, en el segundo apartado se presenta la metodología y las principales fuentes de datos. En la tercera sección se analiza la composición y comportamiento de la economía regional a través de variables e indicadores de uso común. En la cuarta, se analiza la ejecución presupuestal total y de inversiones. En la quinta, se profundiza en la ejecución y administración de la cartera de proyectos. En la sexta, se analizan los sectores sociales de interés: educación y salud. En la séptima, se concluye.

\section{Metodología}

Los estudios que han abordado la performance de un gobierno regional en el Perú son de carácter general y están referidos a la descentralización y regionalización como proceso, como política de Estado. Los sustentos de las conclusiones de los documentos analizados referidos a la marcha del proceso son cualitativos, hechos por actores calificados y observadores especializados, pero se echa en falta un análisis cuantitativo que permita calificar la calidad de la gestión en términos objetivos. En tal sentido, el presente estudio es de carácter exploratorio y descriptivo.

Se ha elegido el desempeño particular de los sectores educación y salud porque son considerados ejes del Índice de Desarrollo Humano (IDH), por el Programa de Naciones Unidas para el Desarrollo (PNUD). Se ha trabajado con datos disponibles en el Portal de Transparencia (Consulta Amigable) y en el banco de proyectos del Ministerio de Economía y Finanzas (MEF).

Se ha utilizado también el Sistema de Información Regional para la Toma de Decisiones (SIRTOD), del Instituto Nacional de Estadística e Informática (INEI). Para el sector 
educación, se ha consultado el sistema Escale del Ministerio de Educación (MINEDU). La información de carácter económico se obtuvo del Banco Central de Reserva del Perú (BCRP). En todos los casos, los pocos errores identificados en los datos y en la clasificación de algunos proyectos fueron corregidos satisfactoriamente.

Se trata de conocer objetivamente la marcha de las variables económicas seleccionadas desde un enfoque descriptivo, dejando para trabajos posteriores la posibilidad de profundizar en algunos aspectos más específicos. Se hace la salvedad que se ha utilizado la información disponible de fuentes oficiales, por lo que las series de tiempo no siempre abarcan el íntegro del período bajo estudio.

\section{La economía regional}

En el presente trabajo, se recurre al valor agregado bruto de producción (VAB) tanto para medir la evolución en el tiempo de la actividad productiva regional (es decir, como tasa de crecimiento) como para medir la contribución de los diversos sectores.

\section{Figura 1}

Perú y Arequipa: crecimiento económico, 2003-2018 (\%)

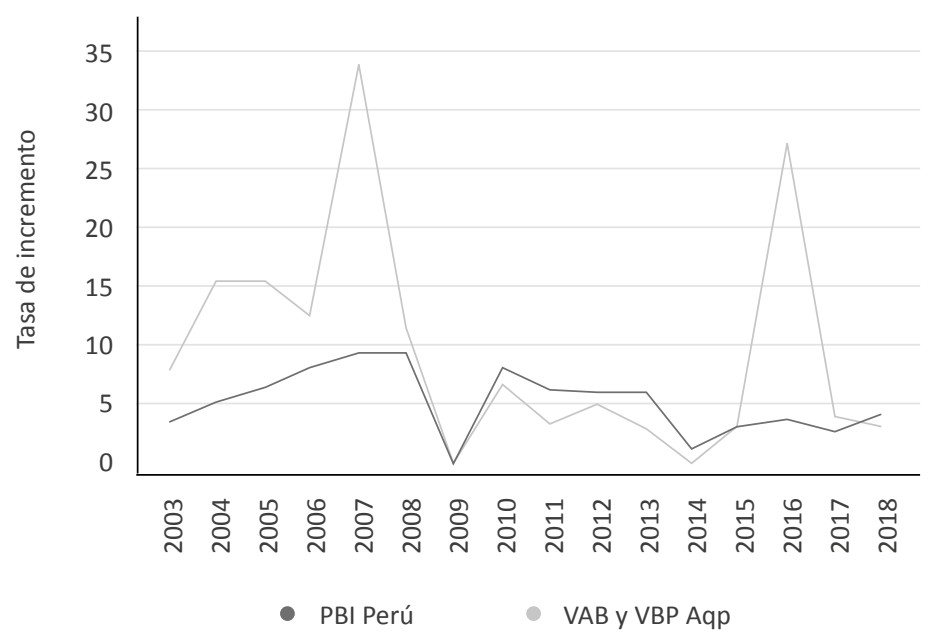

Nota. El crecimiento de Arequipa se mide con el Valor Bruto de la Producción hasta el año 2007. Elaboración propia basada en información del Banco Central de Reserva del Perú (BCRP). 
En el período 2003-2018 (figura 1), con excepción del año 2009, el Perú logró un crecimiento económico importante y sostenido que, según datos del INEI, redujo la pobreza de $33.5 \%$ en el año 2009 a $20.5 \%$ en el 2018 (INEI, 2021), lo que propició el surgimiento de una pujante clase media.

En el caso de Arequipa, puede observarse claros picos de crecimiento, la mayoría de ellos coincidentes con el crecimiento de los volúmenes de producción del sector minero-metálico y los altos precios internacionales del cobre (tabla 1).

\section{Tabla 1}

Períodos de crecimiento extraordinario del sector minería

\begin{tabular}{ccc}
\hline $\begin{array}{c}\text { Años de crecimiento extraordinario } \\
\text { de la región }\end{array}$ & $\begin{array}{c}\text { Tasa anual de crecimiento } \\
\text { económico regional (\%) }\end{array}$ & $\begin{array}{c}\text { Tasa de crecimiento anual del } \\
\text { sector minería (\%) }\end{array}$ \\
\hline 2004 & $15 \%$ & $54 \%$ \\
2005 & $15 \%$ & $34 \%$ \\
2008 & $33.9 \%$ & $198 \%$ \\
2016 & $11.6 \%$ & $23 \%$ \\
\hline
\end{tabular}

Nota. Elaboración propia basada en información del BCRP, Sucursal Arequipa.

El crecimiento de la región depende en gran medida del sector minero, que a su vez genera un movimiento importante en los sectores naturalmente integrados, como el industrial minero-metalúrgico, transportes, servicios varios, entre otros (Apoyo, 2018). En el período 2003-2008, mientras el país creció en promedio a una tasa de $5.25 \%$ anual, la región lo hizo en $9.26 \%$. Si se normalizan los años de excepcional crecimiento minero y se relacionan con la tasa nacional, el crecimiento regional resulta ligeramente menor: $4.98 \%$.

A diferencia del sector minero, que creció de manera continua hasta alcanzar una participación en torno al $37 \%$, el sector público tiene un impacto mucho menor en la actividad económica. Según cifras oficiales, en el período 2007-2018, el sector administración pública contribuye con el $3 \%$ del valor agregado bruto de producción, al cual se le podría añadir lo producido por las empresas públicas de servicios de agua y saneamiento y de distribución de energía, para llegar a una cifra cercana al 5\% (figura 2) 


\section{Figura 2}

Contribución de los principales sectores al VAB de Arequipa, 2007-2018

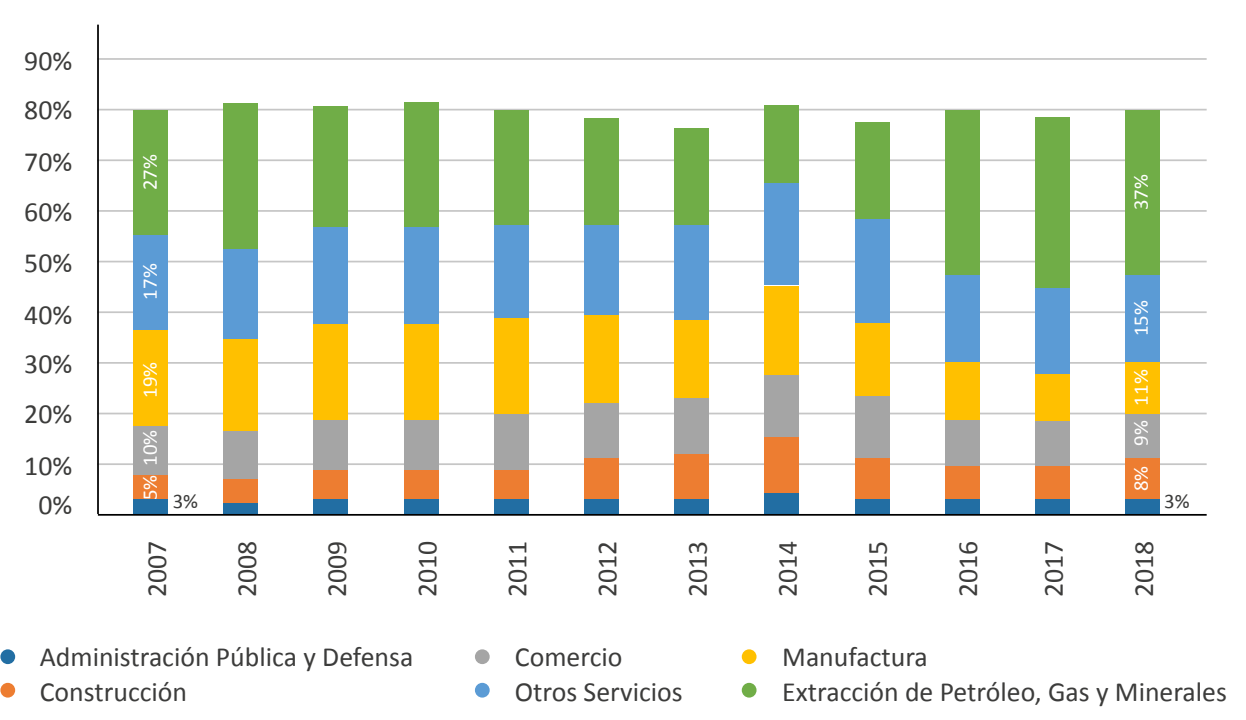

Nota. Elaboración propia basada en información del Banco Central de Reserva del Perú (BCRP).

\section{La ejecución presupuestal del Gobierno Regional de Arequipa (GRA)}

Por presupuesto nos referimos aquí al Presupuesto Institucional Modificado (PIM), que es el presupuesto inicial de apertura (PIA) actualizado con las modificaciones presupuestarias efectuadas durante el año fiscal. En este análisis se excluye el presupuesto de los gobiernos locales (municipalidades provinciales y distritales), cuya labor es eminentemente vecinal. El análisis se hace para el período 2005-2018, que son los años para los cuales se cuenta con información.

En el año 2002, antes del inicio del proceso y la instalación del primer gobierno regional, el departamento de Arequipa, sin considerar gobiernos locales, recibió presupuestos cercanos a los 855 millones de soles anuales. En el 2018 ese presupuesto alcanzó los 4,475 millones de soles (un incremento de $423 \%$ ), manejados en un 55\% por el gobierno regional y en el $45 \%$ restante por el nacional.

En el manejo de los recursos públicos asignados a la región Arequipa, el presupuesto es compartido entre el «nuevo» gobierno regional y el «viejo» gobierno nacional. En la figura 3 se observa que, durante el período de análisis, el gobierno nacional y el gobierno regional 


\section{Figura 3}

Presupuesto total asignado a la región Arequipa por gestores: gobierno nacional y gobierno regional, 2005-2018 (millones de soles y \%)

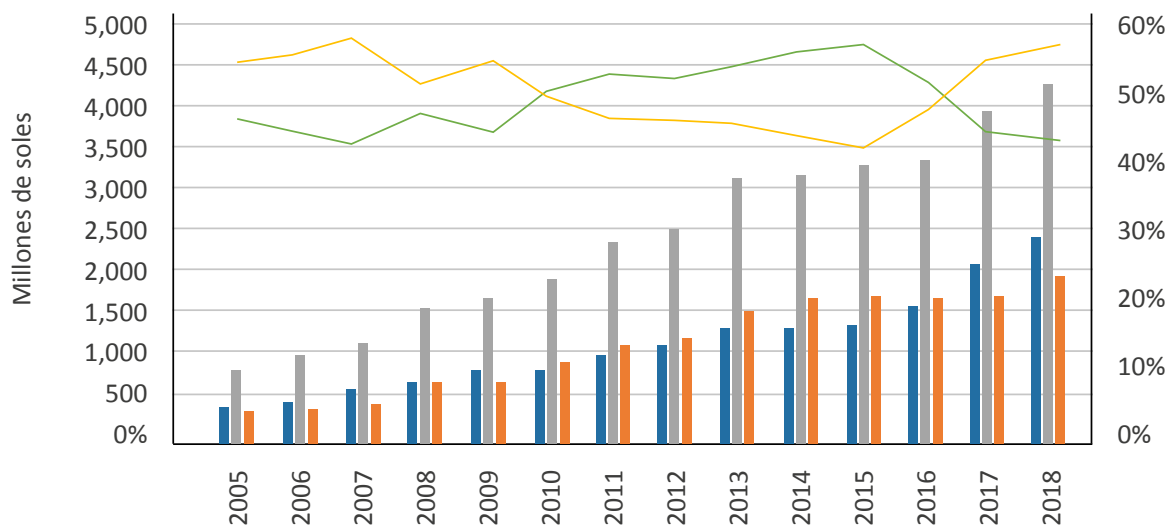

- G. Regional - G. Nacional - Total - G. Regional - G. Nacional

Nota. Elaboración propia con información del Ministerio de Economía y Finanzas (MEF).

\section{Figura 4}

Presupuesto de inversiones asignado a la región Arequipa por gestores: gobierno nacional y gobierno regional, 2005-2018 (millones de soles y \%)

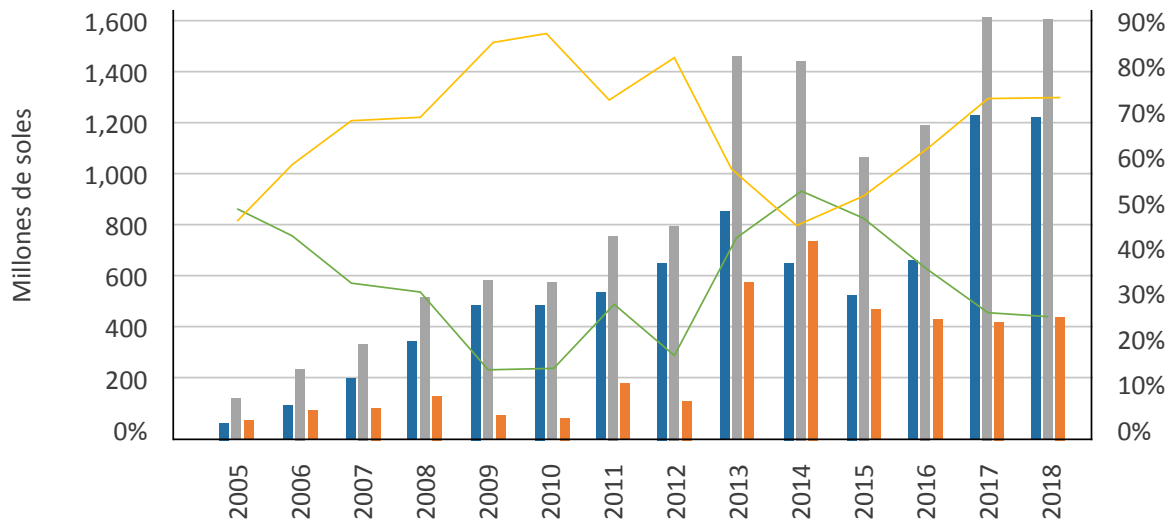

- G. Regional

- G. Nacional

- Total

G. Regional

- G. Nacional

Nota. Elaboración propia con información del Ministerio de Economía y Finanzas (MEF). 
compartieron el manejo presupuestal de la región Arequipa en partes aproximadamente iguales. La regionalización en este sentido ha funcionado, literalmente, «a medias».

En la figura 4 se muestran los presupuestos de inversiones. El crecimiento es también sustancial. De 141 millones de soles recibidos el año 2005, se pasó a 1,435 millones de soles en el 2018, lo cual representa un crecimiento de $917.7 \%$. En el período bajo análisis, se han destinado 10,863 millones a inversiones en todos los sectores. Aquí la situación es diferente en cuanto a la gestión. Si bien el gobierno nacional ha manejado una parte significativa, la participación del gobierno regional es considerablemente mayor, $66 \%$ del total, equivalente a 7,137 millones de soles, habiendo llegado a niveles superiores al 80\% en los años 2009, 2010 y 2012.

Entre ambas instancias de gobierno, en el período se ha asignado a la región 37,596 millones de soles, de los cuales 10,863 fueron para inversiones.

\section{Tabla 2}

Presupuesto del GRA por sectores en el período 2005-2018

\begin{tabular}{|c|c|c|c|c|c|}
\hline Función & PIM & Participación (\%) & Ejecutado & Devuelto & Ejecución (\%) \\
\hline Educación y cultura & $5,513,891,469$ & $29 \%$ & $5,229,302,464$ & $284,589,005$ & $95 \%$ \\
\hline Salud & $4,284,461,476$ & $23 \%$ & $3,783,337,240$ & $501,124,236$ & $88 \%$ \\
\hline Transporte & $2,808,415,866$ & $15 \%$ & $1,947,018,611$ & $861,397,255$ & $69 \%$ \\
\hline Agropecuaria & $1,866,303,122$ & $10 \%$ & $1,097,403,201$ & $768,899,921$ & $59 \%$ \\
\hline Saneamiento & $567,602,077$ & $3 \%$ & $418,305,161$ & $149,296,916$ & $74 \%$ \\
\hline Otros sectores & $506,319,127$ & $3 \%$ & $411,148,270$ & $94,425,921$ & $81 \%$ \\
\hline $\begin{array}{c}\text { Total sectores sociales } \\
\text { y productivos }\end{array}$ & $15,546,993,137$ & $82 \%$ & $12,886,514,947$ & $2,659,733,254$ & $83 \%$ \\
\hline Asistencia social & $560,195,122$ & $3 \%$ & $509,143,404$ & $51,051,718$ & $91 \%$ \\
\hline $\begin{array}{l}\text { Administrativos, } \\
\text { reservas y deuda }\end{array}$ & $1,146,494,066$ & $6 \%$ & $839,796,725$ & $780,581,855$ & $73 \%$ \\
\hline $\begin{array}{l}\text { Previsión social } \\
\text { (pensiones) }\end{array}$ & $1,599,933,111$ & $8 \%$ & $1,585,972,221$ & $13,960,890$ & $99 \%$ \\
\hline Total & $18,853,615,436$ & $100 \%$ & $15,821,427,297$ & $3,505,327,717$ & $84 \%$ \\
\hline
\end{tabular}

Nota. Elaboración propia con información del Ministerio de Economía y Finanzas (MEF). 
Se puede concluir de este primer análisis que, a partir de la creación del gobierno regional, el crecimiento del presupuesto total asignado a la región Arequipa ha sido muy significativo y es administrado en aproximadamente un $50 \%$ por cada nivel de gobierno. En cuanto a inversiones, la tasa de crecimiento ha sido mayor, con una mayor proporción manejada por el GRA.

Este trabajo se centra en la porción de recursos financieros del presupuesto (PIM) del GRA, sin analizar los recursos gestionados por el Gobierno Nacional aplicados en la región. En la tabla 2 se puede observar el presupuesto asignado a la autoridad regional por sectores económicos durante el período en estudio, el mismo que asciende a 18,954 millones, de los cuales el $82 \%$ se asignó a sociales y productivos. Cabe resaltar que 2,660 millones de soles destinados a estos sectores no fueron utilizados.

La responsabilidad de la gestión pública del GRA se concentra en cuatro sectores: los sociales, educación y salud, en un primer grupo, con el 52\% del presupuesto; en segundo nivel de importancia, el sector transportes con $15 \% \mathrm{y}$, finalmente, el agropecuario con $10 \%$ (este último con una alta concentración en el proyecto Majes Siguas II). Se destina un $3 \%$ a saneamiento y $3 \%$ para otros sectores como turismo, industria, pesca, comercio, servicios, etc. El impacto del presupuesto en la producción de bienes y servicios es bajo, porque los fondos se concentran en los servicios sociales básicos y, en menor medida, en infraestructura de transporte. La única actividad productiva que recibe una asignación relevante es la agropecuaria, para la gestión del proyecto Majes Siguas II.

Los sectores sociales tienen una ejecución presupuestal alta, sobre todo educación (con el 95\%), debido al alto contenido de gasto corriente para sueldos y gastos operativos (recursos que deben utilizarse inevitablemente). Transporte, sector clave para dotar de infraestructura productiva a la región, tiene una ejecución de solo el $69 \%$, con proyectos importantes que se encuentran inconclusos o paralizados.

Por otro lado, existen presupuestos muy bajos para otros sectores en los que el GRA participa con una generación de poco impacto, ciertas ineficiencias y problemas de planificación (véase tabla 3 ).

Los presupuestos exiguos para estos «otros» sectores sugieren que los planes pueden convertirse en un listado de buenas intenciones, incoherentes 


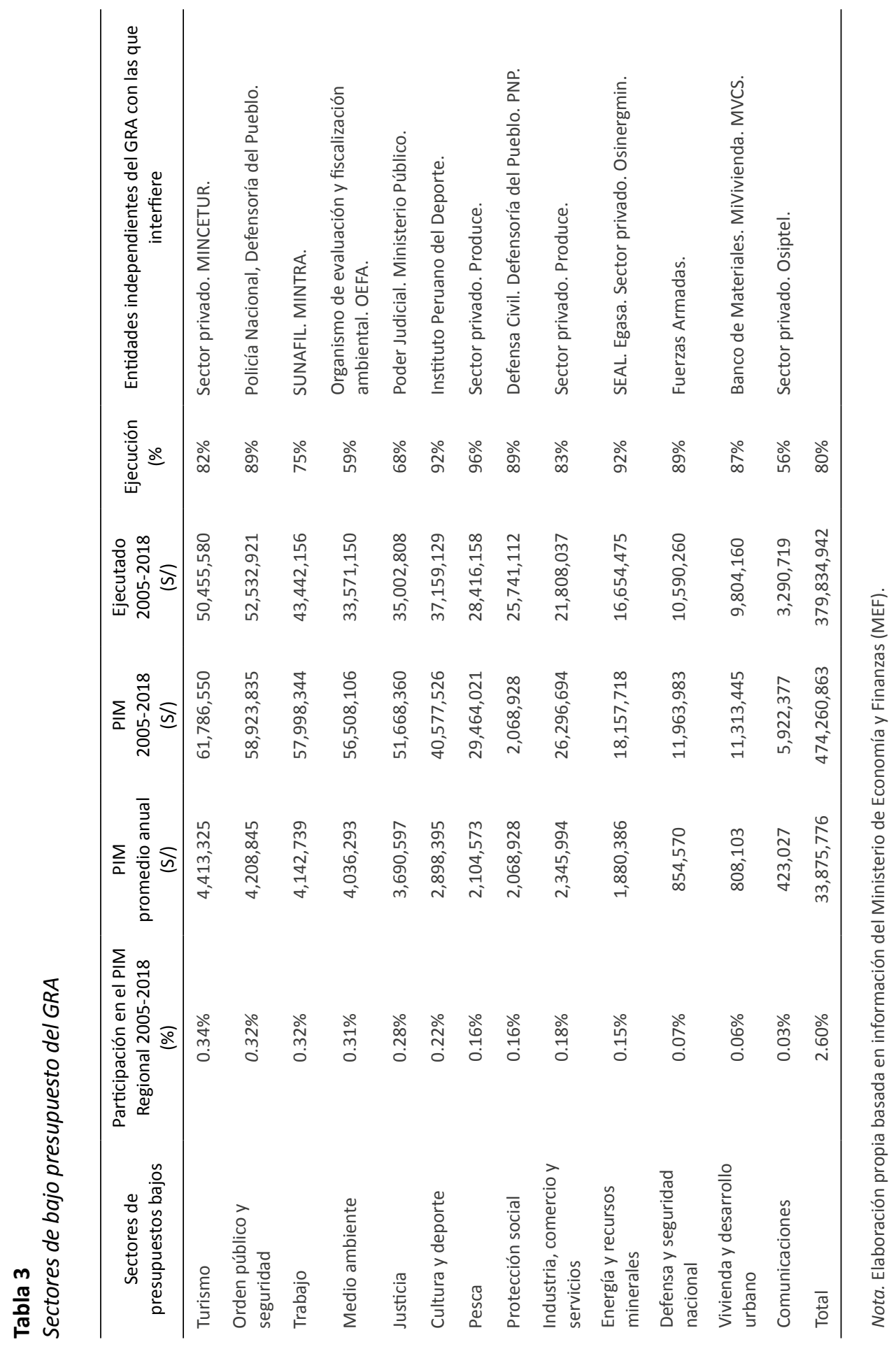


y puramente declarativos, con el potencial de distraer al GRA de los objetivos de los sectores a los que orienta la mayor parte de su presupuesto (educación y cultura, salud, transporte, agropecuaria, saneamiento).

La figura 5 muestra el incremento sustancial de los montos asignados al GRA. Los presupuestos per cápita pasaron de 672 soles en el 2007 a 1,789 soles en el 2017. El gobierno nacional también ha mostrado un incremento (véase la figura 4), por lo que no sería correcto proponer que el crecimiento obedece a que el dinero pasó de una instancia administrativa a otra: el incremento fue real. Más relevante aún es el incremento continuo del presupuesto de inversiones asignado al GRA, que pasó de 68 millones de soles a 1,043 millones de soles.

\section{Figura 5}

Evolución del presupuesto anual total y de inversiones del GRA, 2005-2018 (millones de soles corrientes)

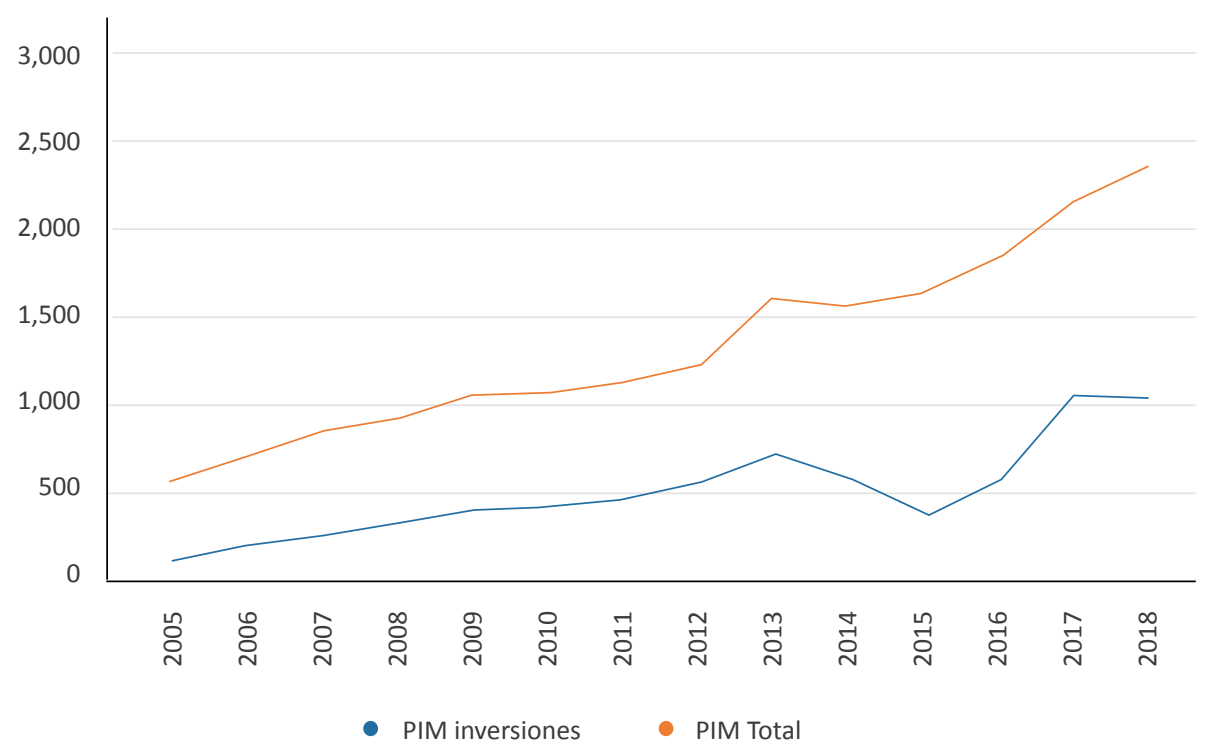

Nota. Elaboración propia basada en información del Ministerio de Economía y Finanzas (MEF).

En el presupuesto del GRA hay un alto componente de gasto corriente: bienes y servicios y remuneraciones de maestros y personal de salud, a lo que se suma la planilla de trabajadores de la propia entidad y otros gastos corrientes menores. En el caso del GRA, la proporción destinada a inversiones inició con un $12 \%$ del total, con una tendencia creciente, llegando al $42 \%$ en el 2018 (véase la figura 6). 


\section{Figura 6}

Presupuesto del GRA: gasto corriente y de inversión, 2005-2018 (estructura porcentual)

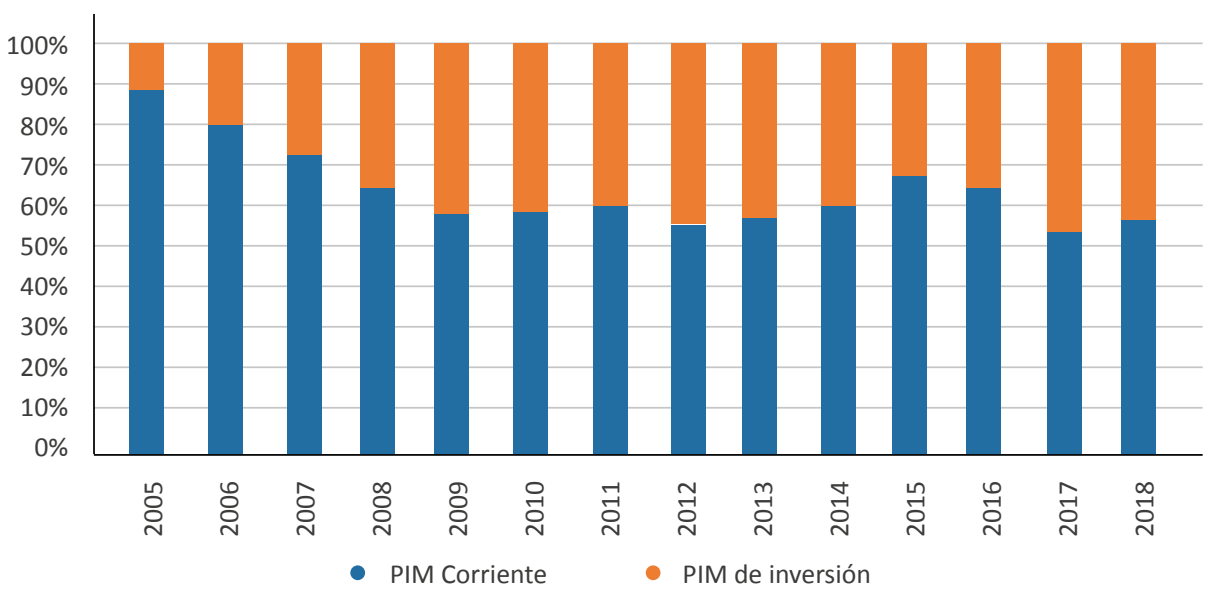

Nota. Elaboración propia basada en información del Ministerio de Economía y Finanzas (MEF).

\section{Figura 7}

Evolución de la ejecución presupuestal, 2005-2018 (\%)

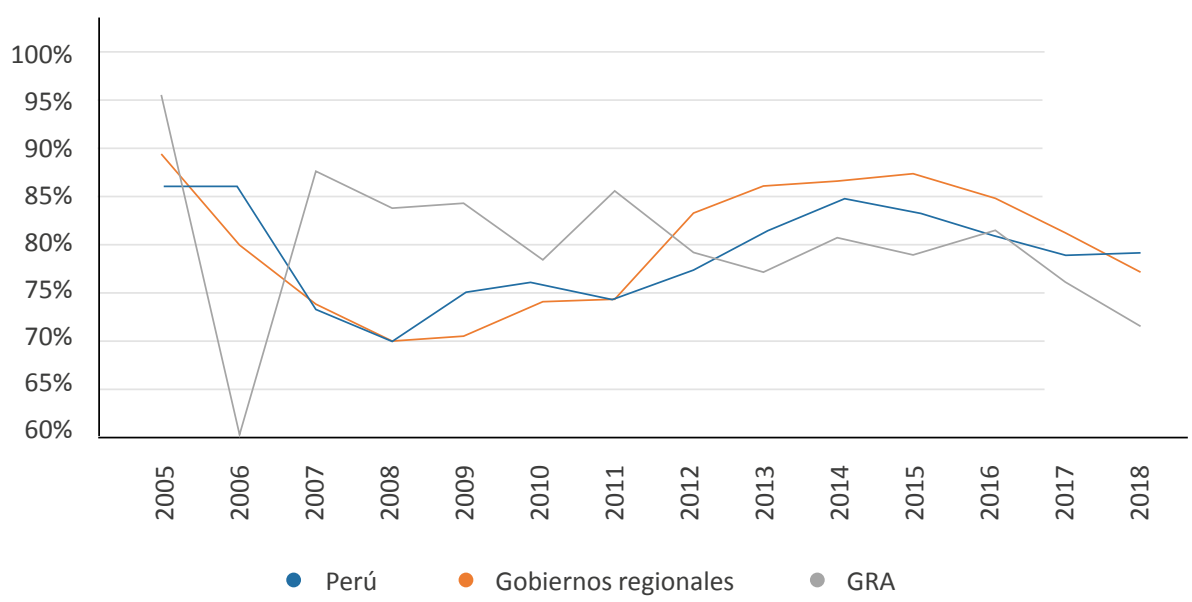

Nota. Elaboración propia basada en información del Ministerio de Economía y Finanzas (MEF). 
En el análisis de la ejecución presupuestal del dinero asignado solamente al GRA, cabe advertir que estas cifras consideran las transferencias realizadas a otras entidades públicas, como municipalidades, que podrían no haber sido utilizadas, de manera que podrían seguir sin generar bienes y servicios públicos. En la figura 7 se muestra la ejecución del GRA, comparándola con la del conjunto de regiones y el total del presupuesto nacional.

La ejecución del presupuesto total asignado al GRA presenta altibajos. Desde el 2005 hasta el 2016 se mantuvo por encima del 85\%, con excepción del año 2006, en el que bajó a 70\%. En el 2018 cayó a 80\%. Se observa que, a partir del 2013, la ejecución presupuestal está por debajo de la del presupuesto nacional y de las demás regiones en conjunto.

En la figura 8 se muestra la ejecución del presupuesto de inversiones; estas podrían tener un efecto positivo sobre la capacidad productiva regional, es decir, sobre el crecimiento y potencial desarrollo, de una manera mucho más directa que el gasto corriente. La tasa de ejecución disminuyó hasta llegar a su nivel más bajo, 58\%, en el 2018.

En el año 2018, el déficit acumulado de ejecución del presupuesto de inversiones de la región Arequipa llegó a los 2,260 millones de soles (véase la figura 9).

\section{Figura 8}

Ejecución presupuestal de inversiones del Gobierno Regional de Arequipa, 2005-2018 (\%)

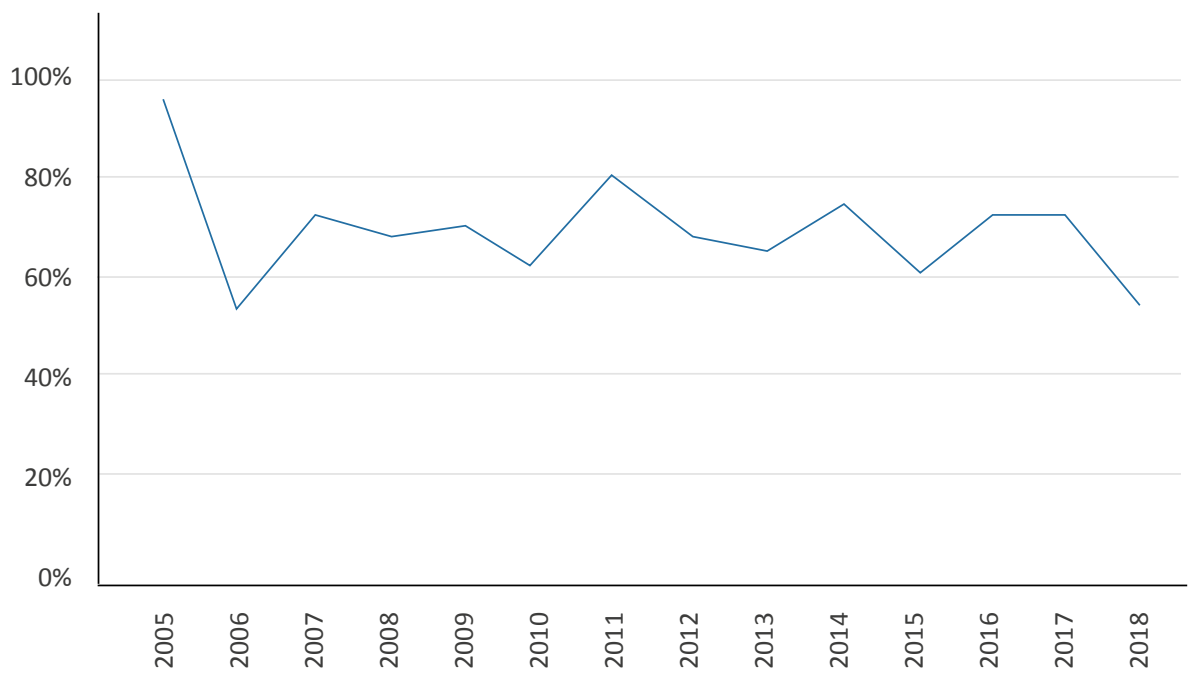




\section{Figura 9}

Déficit de ejecución de inversiones del presupuesto anual y acumulado del GRA, 2005-2018 (millones de soles)

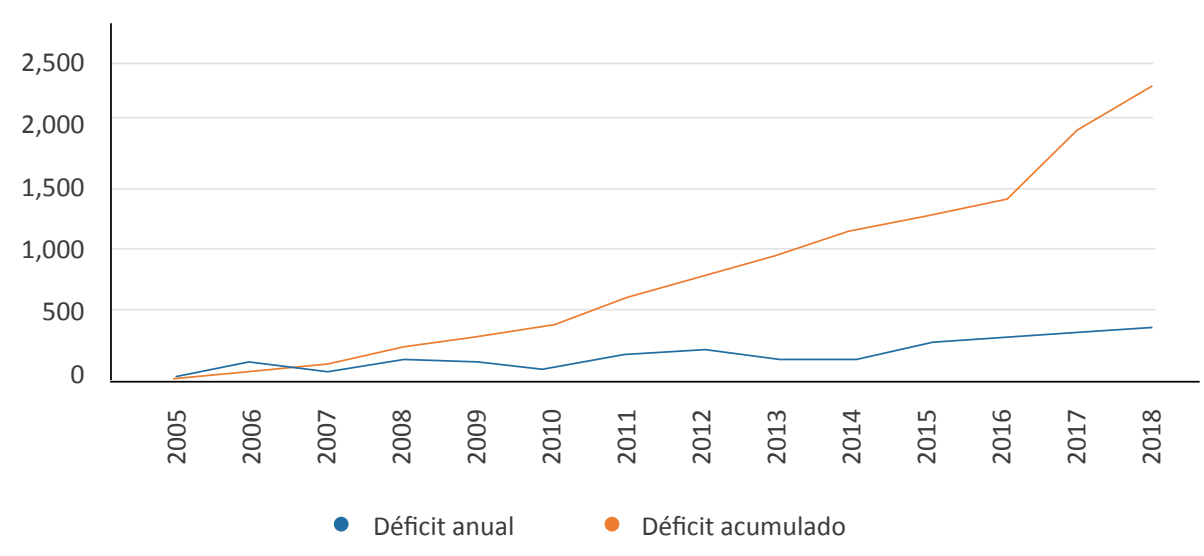

Nota. Elaboración propia basada en información del Ministerio de Economía y Finanzas (MEF).

\section{La administración de los proyectos de desarrollo}

Las inversiones se materializan a través de proyectos. En el Perú, la norma vigente en el momento del análisis es la Ley N. ${ }^{\circ} 27293$, del Sistema Nacional de Inversión Pública (SNIP), el que «tiene como finalidad optimizar el uso de los recursos públicos destinados a la inversión». El MEF define como proyecto viable a aquel cuyo perfil o estudio de preinversión haya demostrado ser socialmente rentable, sostenible y compatible con las políticas sectoriales nacionales o locales (MEF DNPP s/f). Cabe anotar que este sistema cambió de denominación a Invierte.pe, con algunas modificaciones formales.

Haciendo un análisis de la cartera de proyectos viables puede hacerse una aproximación a las prioridades de desarrollo y a la calidad de la gestión de inversiones del GRA.

En el período en análisis, 2005-2018, el GRA dispuso de una cartera total de 1,876 proyectos valorizados en 8,331 millones de soles, de los cuales se ejecutaron solamente 4,439 millones, es decir, el $53.3 \%$ sobre el monto declarado viable, y $42.9 \%$ sobre el monto viable actualizado. Es decir, se dejaron de ejecutar 3,892 millones en proyectos que contaban con viabilidad de parte del SNIP. Cabe advertir que la diferencia entre ese monto y los 2,260 millones del déficit de inversiones acumulado del gráfico 10 corresponden a proyectos que, contando con viabilidad, no se consideraron en ninguno de los presupuestos de los años 2005 al 2018, esto es, un total de 1,645 millones de soles en proyectos viables que se abandonaron; ello muestra, también, falta de eficacia entre el trabajo de preinversión, es decir, de elaboración y trámite de viabilidad de los proyectos. 


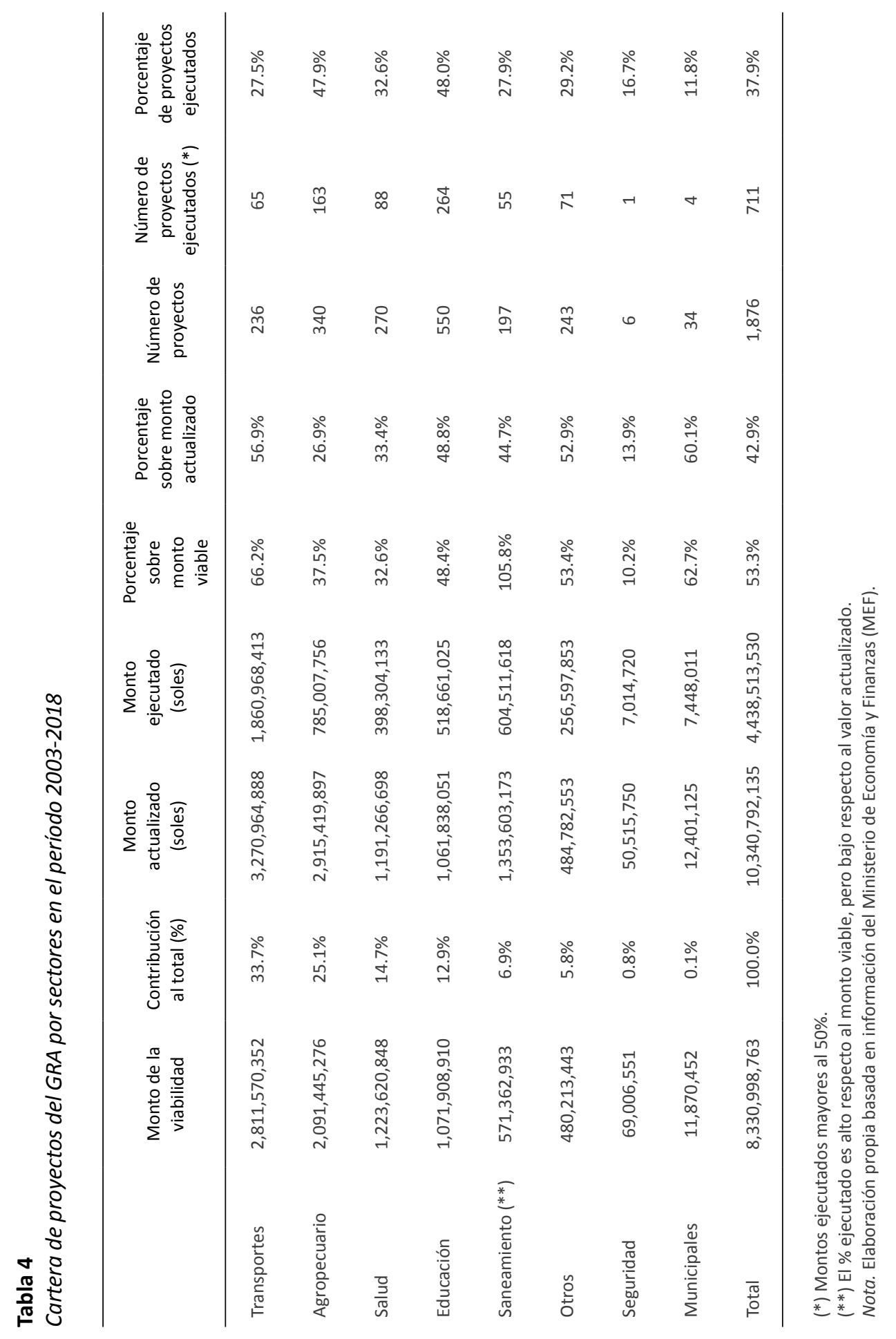


El GRA siempre contó con proyectos en fase de preinversión que podía ejecutar, pero algunos no fueron considerados en los presupuestos anuales de inversión y otros, presupuestados, no se ejecutaron o se ejecutaron parcialmente.

Destaca el peso de los sectores transportes y agropecuario, que concentran el $58.8 \%$ de la cartera de proyectos, con una relativamente alta tasa de ejecución, sobre todo de transportes. El sector salud solo representa el $14.7 \%$ y educación el $12.9 \%$, con niveles de ejecución de algo más del $30 \%$ y del $48 \%$, respectivamente. Los sectores sociales claves fueron relegados por obras de infraestructura, que podrían haber sido manejadas por el gobierno nacional por su grado de complejidad; las autoridades regionales, al encontrarse más cerca de la realidad social, hubieran desempeñado un rol más eficaz en los proyectos de los sectores sociales.

Llama la atención el involucramiento del Gobierno Regional en proyectos de Agua y Saneamiento (6.9\% del total), existiendo una empresa especializada como la Empresa Pública de Servicios de Agua y Saneamiento de Arequipa (EPS - SEDAPAR), de alcance regional. Esta duplicidad genera conflictos al interior del sector y problemas técnicos de concepción de los proyectos, lo que trae como consecuencia la no recepción de las obras por parte de la empresa especialista, así como errores y omisiones en el cálculo de las tarifas al usuario, lo que desordena la gestión y dificulta medir la eficiencia de este servicio fundamental.

En la tabla 5 se ve la cartera de proyectos del GRA, desagregada según lo que el SNIP considera «Estado de la inversión».

\section{Tabla 5}

Cartera de proyectos del GRA entre 2003 y 2018 por estado de la inversión

\begin{tabular}{ccccccc}
\hline $\begin{array}{c}\text { Estado de la } \\
\text { inversión }\end{array}$ & Número & $\begin{array}{c}\text { Porcentaje } \\
\text { sobre el total }\end{array}$ & $\begin{array}{c}\text { Monto viable } \\
\text { (soles) }\end{array}$ & $\begin{array}{c}\text { Porcentaje } \\
\text { sobre el total }\end{array}$ & $\begin{array}{c}\text { Monto ejecutado } \\
\text { (soles) }\end{array}$ & $\begin{array}{c}\text { Tasa de } \\
\text { ejecución } \\
\text { (\%) }\end{array}$ \\
\hline Activos & 872 & $46.5 \%$ & $7,290,725,299$ & $87.5 \%$ & $3,978,369,226$ & $54.6 \%$ \\
Cerrados & 291 & $15.5 \%$ & $596,396,068$ & $7.2 \%$ & $446,006,407$ & $74.8 \%$ \\
$\begin{array}{c}\text { Desactivado } \\
\text { permanente }\end{array}$ & 709 & $37.8 \%$ & $442,717,137$ & $5.3 \%$ & $2,015,218$ & $0.5 \%$ \\
$\begin{array}{c}\text { Desactivado } \\
\text { temporal }\end{array}$ & 4 & $0.2 \%$ & $1,160,260$ & $0.0 \%$ & 46,030 & $4.0 \%$ \\
\multicolumn{1}{c}{ Total } & 1,876 & $100.0 \%$ & $8,330,998,763$ & $100.0 \%$ & $4,426,436,881$ \\
\hline
\end{tabular}

Nota. Elaboración propia basada en información del Ministerio de Economía y Finanzas (MEF). 
En este cuadro se aprecia que, durante el período 2003-2018, existieron 1,876 proyectos declarados viables, por 8,331 millones de soles, de los cuales $46.5 \%$ permanecieron activos, que podrían haber constituido inversión por un monto de 7,290 millones de soles, de los cuales se ejecutó solamente el $54.6 \%$.

Prácticamente ningún proyecto de inversión de envergadura ejecutado por el Gobierno Regional se ha culminado satisfactoriamente; solo uno, ejecutado en su primera etapa por privados bajo el programa «Obras por impuestos», se puede decir que está ejecutado al $98 \%$ y en operación satisfactoriamente.

\section{Tabla 6}

Situación de los principales proyectos de inversión del GRA al 2018

\begin{tabular}{|c|c|c|c|c|c|}
\hline Nombre de la inversión & $\begin{array}{l}\text { Monto viable } \\
\text { (soles) }\end{array}$ & Función & $\begin{array}{l}\text { Fecha de } \\
\text { viabilidad }\end{array}$ & $\begin{array}{l}\text { Tasa de } \\
\text { ejecución } \\
(\%)\end{array}$ & Observaciones \\
\hline Majes Siguas - II Etapa. & $1,338,419,640$ & Agraria & $10 / 01 / 2008$ & $40.1 \%$ & Paralizado. \\
\hline $\begin{array}{l}\text { Construcción de la vía } \\
\text { regional Arequipa - La Joya, } \\
\text { en las progresivas km 0+00 al } \\
\text { km } 24+540 \text {, distritos de Cerro } \\
\text { Colorado - La Joya. }\end{array}$ & $590,385,602$ & Transporte & $25 / 01 / 2016$ & $14.9 \%$ & Paralizado. \\
\hline $\begin{array}{l}\text { Ampliación y mejoramiento } \\
\text { de los servicios de salud del } \\
\text { Hospital Goyeneche, nivel III - } \\
\text { 1, del distrito de Arequipa. }\end{array}$ & $520,393,430$ & $\begin{array}{c}\text { Salud y } \\
\text { Saneamiento }\end{array}$ & $01 / 08 / 2017$ & $0.7 \%$ & Paralizado. \\
\hline $\begin{array}{l}\text { Construcción de vía troncal } \\
\text { interconectora entre los } \\
\text { distritos de Miraflores, Alto } \\
\text { Selva Alegre, Yanahuara, } \\
\text { Cayma y Cerro Colorado de la } \\
\text { provincia de Arequipa. }\end{array}$ & $369,518,316$ & Transporte & $31 / 03 / 2009$ & $121.7 \%$ & $\begin{array}{l}22 \% \text { de costo } \\
\text { adicional. Obras } \\
\text { por impuestos, } \\
\text { al } 98 \% \text { de } \\
\text { ejecución, en } \\
\text { operación. }\end{array}$ \\
\hline $\begin{array}{l}\text { Mejoramiento del servicio } \\
\text { de transitabilidad del eje de } \\
\text { integración vial norte entre } \\
\text { la intersección de la Av. Las } \\
\text { Torres - vía PE-34A, hasta la } \\
\text { intersección con la Av. Italia - } \\
\text { Av. Aviación, distritos de Yura } \\
\text { y Cerro Colorado, provincia de } \\
\text { Arequipa, región Arequipa. }\end{array}$ & $266,710,991$ & Transporte & 09/05/2017 & $40.1 \%$ & $\begin{array}{l}\text { En ejecución. } \\
\text { Operación } \\
\text { prevista para el } \\
2021 .\end{array}$ \\
\hline $\begin{array}{l}\text { Mejoramiento de la carretera } \\
\text { Viscachani-Callalli-Sibayo- } \\
\text { Caylloma, provincia Caylloma. }\end{array}$ & $256,441,254$ & Transporte & 23/06/2015 & $33.3 \%$ & $\begin{array}{l}\text { En ejecución. } \\
\text { Retrasado cuatro } \\
\text { años. }\end{array}$ \\
\hline
\end{tabular}


Mejoramiento y ampliación

de la frontera agrícola

optimizando los recursos

$254,489,067$

Agropecuaria

$27 / 05 / 2016$

$15.1 \%$

$52 \%$ de costo

hídricos de la subcuenca del

río Arma, Condesuyos.

Construcción de la autopista

regional Arequipa - La Joya.

\section{8,739,646 Transporte 20/08/2009 46.1\%}

Cerrado

inconcluso.

Infraestructura

abandonada.

Mejoramiento de la carretera Variante de Uchumayo, entre el puente San Isidro y la Vía de Evitamiento, distritos Sachaca, Yanahuara y Cerro Colorado, provincia de Arequipa.

$214,432,065$

Transporte

06/06/2013

$263.7 \%$

$293 \%$ de costo adicional. En operación, no culminado. Infraestructura $\sin$ mantenimiento.

Mejoramiento de la transitabilidad en la vía departamental, ruta AR-109 en los distritos de Majes, Lluta y Huambo de la provincia de Caylloma.

Nota. Elaboración propia basada en información del Ministerio de Economía y Finanzas (MEF).

No obstante, en el Análisis de Sostenibilidad de la Alternativa Recomendada que presentan los funcionarios para aprobación de los proyectos de inversión, se continúa afirmando que: «El presente proyecto es sostenible porque cumple con las condiciones requeridas: Marco Normativo que respalda su ejecución; arreglos institucionales; las instituciones involucradas cuentan con capacidad de gestión que se sustenta en el equipo humano altamente calificado que forma parte de su estructura orgánica, así como la experiencia necesaria en proyectos de este tipo» ${ }^{1}$. Nada más lejano a la realidad, a la luz de los resultados de la ejecución de proyectos.

La situación se hace algo confusa cuando se ven 291 proyectos cerrados por 596 millones, representando el $15.5 \%$ del total de proyectos viables. Si se siguiera la norma, se debieran esperar proyectos culminados, es decir, con el gasto del $100 \%$ de los recursos asignados. Sin embargo, se observa que solo se gastaron 446 millones, equivalentes al 74.8\%. Este resultado sugiere que parte de los proyectos declarados como «cerrados» no están terminados o culminados.

En la tabla 7 se muestran los proyectos cerrados por la porción de la inversión ejecutada, representada por el monto devengado acumulado, respecto al $100 \%$ presupuestado en la viabilidad, así como también respecto del monto de inversión actualizado que, generalmente, es mayor — producto de ajustes sucesivos- - . De esta manera, se puede tener una aproximación para saber si los proyectos fueron cerrados a su culminación. 


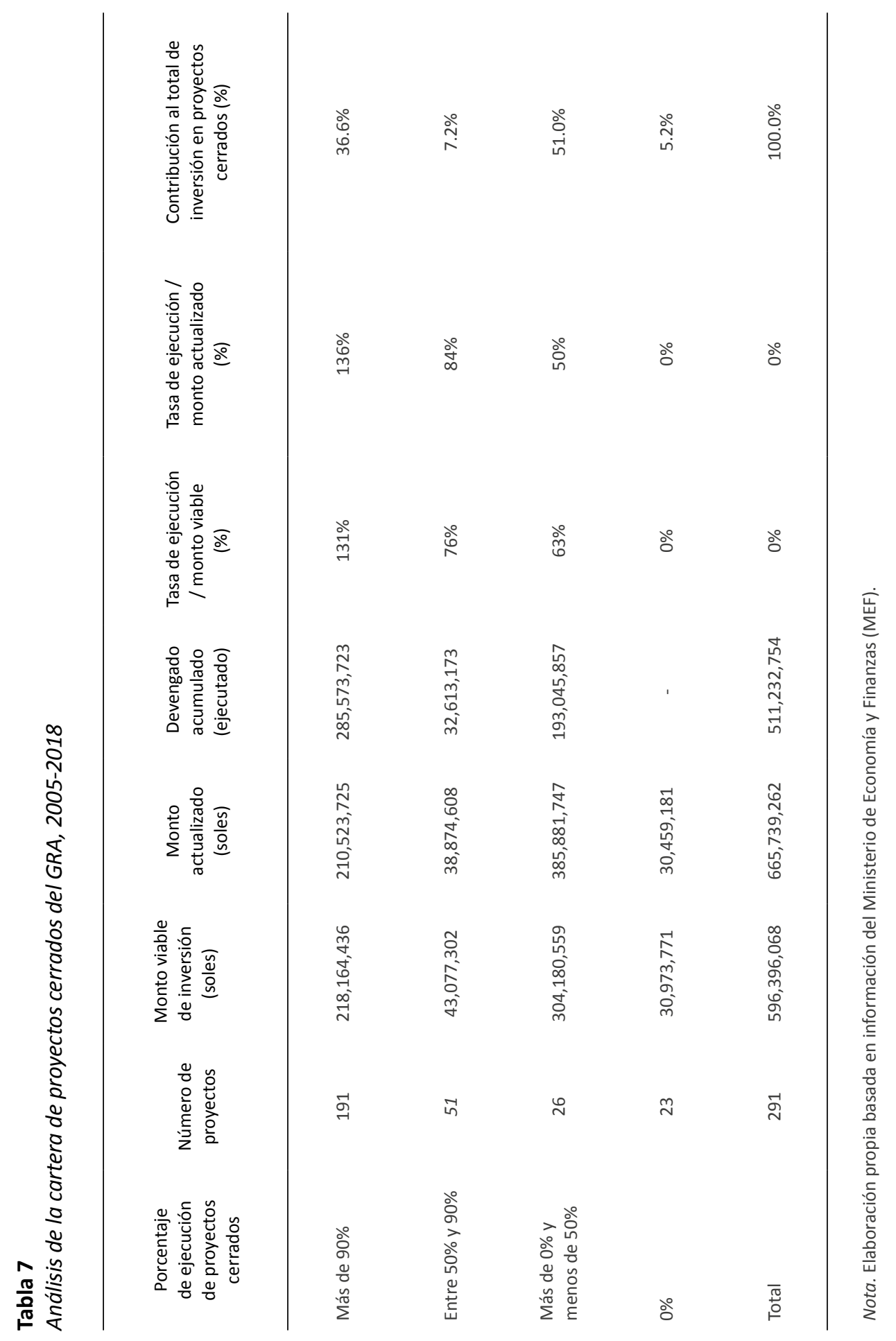


Tabla 8

Proyectos cerrados con inversión inferior al 90\% del monto viable, 2005-2018

Nombre de la inversión
Monto viable Función

Porcentaje de ejecución al cierre del proyecto
Construcción de la autopista regional Arequipa - La Joya.

Mejoramiento de los servicios educativos en la I.E. Pinto Talavera, AA.HH. villa confraternidad, zona b - distrito de Alto Selva Alegre.

Mejoramiento de los servicios en salud oncológica del Departamento de Oncología y Radioterapia del Hospital III-1 Goyeneche.

Mejoramiento del servicio educativo en la I.E. Arequipa.

Mejoramiento de la carretera Chivay - Tuti, Caylloma.

Mejoramiento de los servicios educativos de la i.e. n. 040616 - Casimiro Cuadros del distrito de Cayma.

Instalación del sistema de agua potable y alcantarillado sanitario para 07 asentamientos humanos en el sector Umapalca, distrito de Sabandía.

Mejoramiento del servicio educativo en el colegio diversificado industrial politécnico nacional de Arequipa, Rafael Santiago Loayza Guevara, en el distrito de Mariano Melgar.

Mejoramiento bocatoma y canal plataforma valle de Tambo, distrito de Cocachacra, Islay.

Mejoramiento vía departamental D-118, tramo cruce Chaviña - cruce Medina, Caravelí.

Mejoramiento de la capacidad resolutiva del centro de salud Cabanaconde, distrito de Cabanaconde, provincia de Caylloma.
248,739,646 Transporte

$46.1 \%$

$17,244,287$

Educación

$0.0 \%$

$6,379,313$

Salud

$21.7 \%$

$5,948,473$

Educación

$45.9 \%$

5,256,935

Transporte

$63.5 \%$

4,801,952 Educación 39.0\%

4,756,865 Salud y

Saneamiento

4,588,252 Educación 88.2\%

3,692,069 Agropecuaria 43.1\%

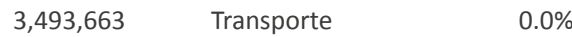

$\begin{array}{ll}3,331,233 & \text { Salud } \\ \end{array}$

Nota. Elaboración propia basada en información del Ministerio de Economía y Finanzas (MEF). 
Sorprende el cierre de 26 proyectos, que representan un monto viable de más de 304 millones, con una ejecución menor al $50 \%$ del monto de inversión actualizado. Este grupo representa el 51\% del monto de proyectos cerrados. Se puede concluir que el cierre de proyectos, en una importante proporción, se hizo con una ejecución menor al 50\%, es decir, proyectos no culminados. Otro indicador que confirma la deficiente ejecución de inversiones por parte de esta instancia de gobierno.

Parte significativa de los principales proyectos cerrados, sin uso total de su presupuesto, pertenece al sector educación, por casi 32.6 millones de soles. Otro proyecto importante cerrado con solo $21.7 \%$ de ejecución pertenece al sector salud. Estos son indicadores del desenfoque estratégico del Gobierno Regional de Arequipa (GRA).

\section{La gestión de los sectores sociales}

La educación es un factor clave para el desarrollo. Está claro que la pobreza cultural y educativa es una traba mayor al desarrollo que la estructural o la carencia de recursos naturales. La salud es preocupación prioritaria porque determina la calidad de vida de las personas.

Sin embargo, estos sectores sociales no son foco de atención del GRA, pese a que el $63 \%$ del presupuesto total es destinado a ellos. En las figuras 10 y 11 se observa el destino de los presupuestos de educación y salud. Se observa que el segmento dedicado a inversiones, es decir, a mejorar la capacidad productiva de los servicios, es limitada, aun así, no logra ejecutarse en su integridad, como se verá más adelante.
Figura 10

Presupuesto de Educación del GRA, 2005-2018

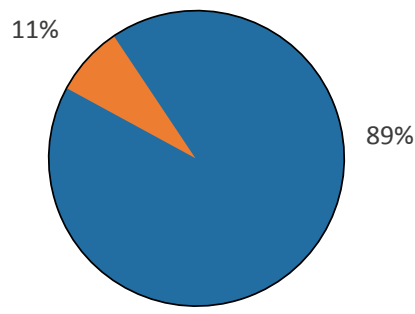

- Gasto corriente: S/ 4,554,158,901

- Inversión: S/ 590,269,937

Nota. Elaboración propia basada en información del Ministerio de Economía y Finanzas (MEF).

Figura 11

Presupuesto de salud del GRA, 2005-2018

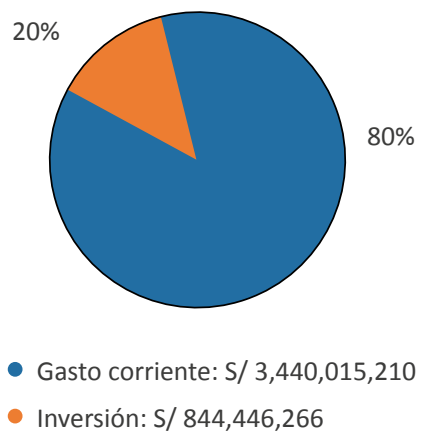

Nota. Elaboración propia basada en información del Ministerio de Economía y Finanzas (MEF). 


\subsection{El sector educación}

Una primera interrogante respecto a la gestión del GRA, en el sector educación, es si sus planes estratégicos se encuentran alineados, dado que recibe la mayor parte de los recursos presupuestales. En el primer plan de desarrollo del año 2003 no existen estrategias ni objetivos específicos para el sector educación. Los seis grandes ejes estratégicos se refieren a dinamizar la economía y desarrollarla productivamente priorizando la agroindustria de exportación, ser un destino turístico competitivo, sostener el medio ambiente, fortalecer la identidad ciudadana y calificarla, y promover la participación del sector privado (GRA, 2003).

En el diagnóstico del último plan, que data del año 2015, la mención a los resultados de la Evaluación Censal de Estudiantes (ECE) del MINEDU, como indicador de la calidad educativa, hace hincapié en la mejora reciente y no plantea metas que cierren las brechas existentes. Como causa de las deficiencias se señala: los bajos sueldos de los profesores (los que responden a la Ley de la Carrera Pública Magisterial, sobre lo que el GRA poco o nada puede hacer), las limitadas estrategias educativas, la desmotivación de los docentes, los limitados materiales educativos y la mejora de la educación rural (GRA, 2015). No se ven estrategias ni acciones en la línea del diagnóstico. Los objetivos estratégicos sectoriales son demasiado generales, tendrían que ser más específicos en la línea de las debilidades o deficiencias encontradas, que ataquen directamente los problemas de motivación docente, materiales de enseñanza, mejoras logísticas y de la educación rural, entre otros. Tampoco se presenta una propuesta para el trabajo conjunto entre el gobierno nacional y los gobiernos locales. Los planes para el sector educación, a pesar de ser el de mayor presupuesto, tienen serias deficiencias.

En la figura 12 puede verse el importante crecimiento del presupuesto, que pasó de algo más de 200 millones a cerca de 600 millones de soles, es decir, el triple. Es importante también insistir en que la mayor proporción fue destinada a gasto corriente, lo que permitió la contratación de un mayor número de docentes, sobre todo en el nivel inicial (que pasaron de 726 a 1,665) y también, aunque en menor medida, en el nivel secundario. Esto no condice con la reducción del número de estudiantes de primaria y secundaria en instituciones públicas de la región (figura 19).

En cuanto a la ejecución presupuestal, en la figura 13 se muestra un cumplimiento de alrededor del $95 \%$ del presupuesto total, influenciado por el componente de remuneraciones y gasto corriente, con excepción del año 2006, en que la ejecución cae a menos del 60\%. 
Figura 12

Estructura del presupuesto anual de educación del GRA, 2005-2018 (millones de soles)

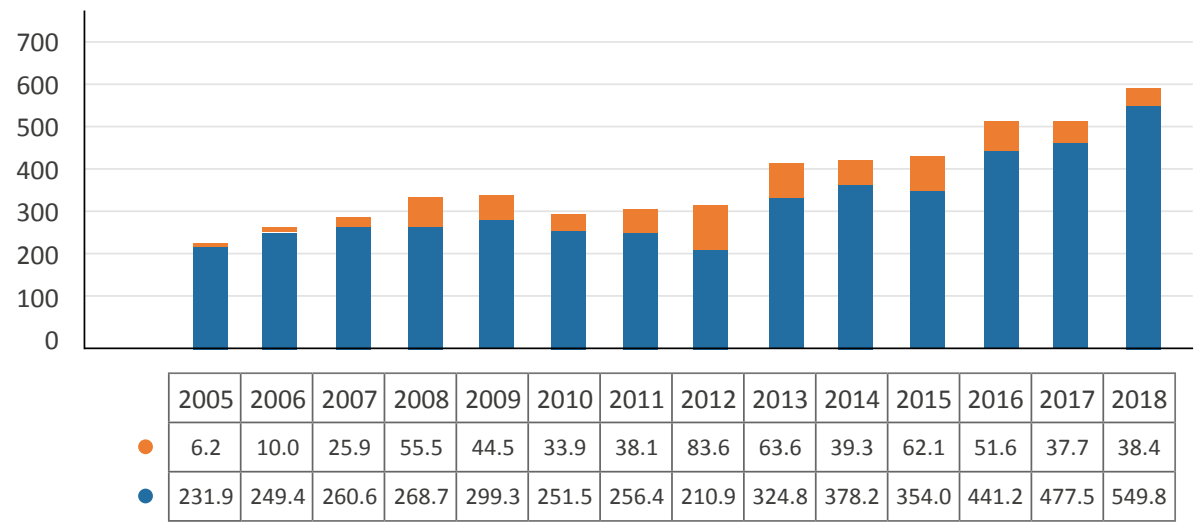

- Gasto corriente Inversión

Nota. Elaboración propia basada en información del Ministerio de Economía y Finanzas (MEF).

\section{Figura 13}

Evolución del presupuesto de educación y ejecución presupuestal, 2005-2018 (millones de soles y \%)

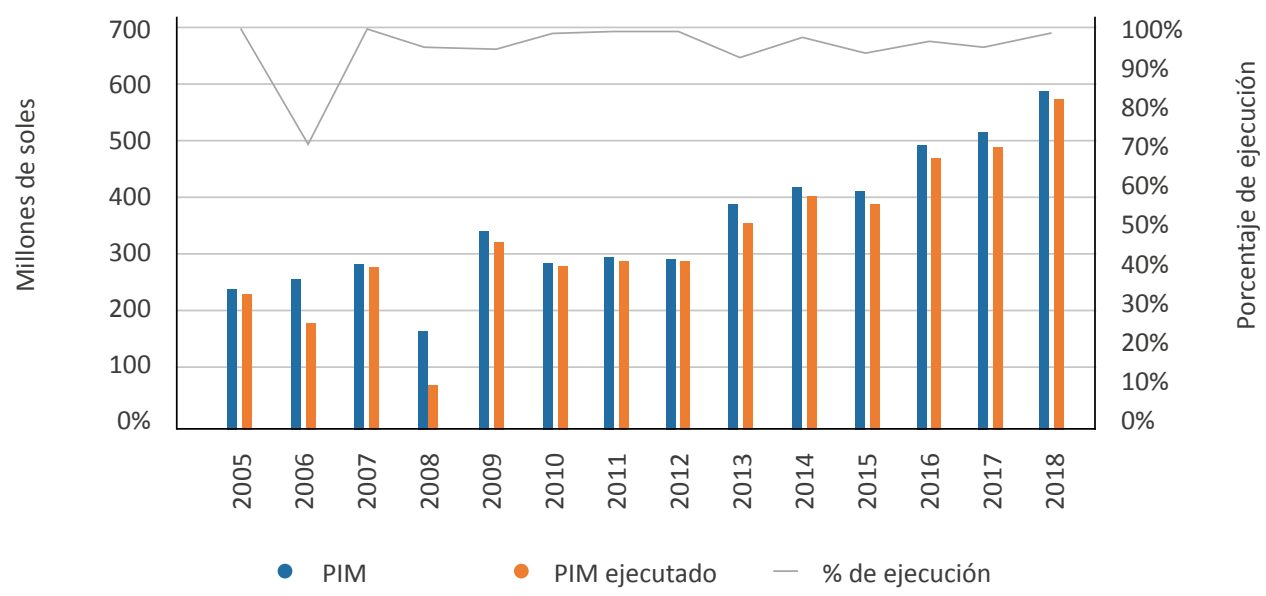

Nota. Elaboración propia basada en información del Ministerio de Economía y Finanzas (MEF). 
En cuanto al presupuesto de inversiones (figura 14), es claro el crecimiento de los montos asignados, que pasan de 6 millones de soles en el 2005, a más de 80 millones en el 2012, para volver a niveles inferiores a los 40 millones en los años 2017 y 2018. Llama la atención el importante volumen de recursos asignados y no utilizados durante el período 2012-2015. Los recursos públicos tienen un alto costo de oportunidad, sobre todo en los servicios básicos; mientras que la mala elaboración de presupuestos o la escasa capacidad de gestión en una región deja sin recursos a otra que podría utilizarlos mejor.

En la figura 15 se aprecian los déficits de ejecución presupuestal de inversiones del sector educación y el acumulado del período, con un pico de 28.4 millones de soles sin utilizar en el año 2012, y de 22.9 y 22.5 millones en los años 2014 y 2016, respectivamente. Se observa una mayor ejecución durante el período 2009-2011. Así, se llega a un total no ejecutado de 136.4 millones de soles.

En la figura 16 se observa que, mientras que el presupuesto destinado a la adquisición de bienes y servicios (rubro en el cual se encuentran los gastos de mantenimiento de activos y compra de servicios) crece, la proporción de instituciones educativas (IE) en buen estado es cada vez menor. En ningún momento se llegó a tener el 50\% de colegios en buen estado (el máximo alcanzado fue $47 \%$ en el 2009), habiéndose llegado al 24\% en el 2015 y $30 \%$ en el año 2018 (un gran perjuicio para los niños y jóvenes que se forman en ambientes inadecuados y que podrían exponerlos al peligro).

En la figura 17 se observa que el porcentaje de instituciones educativas con tres servicios básicos crece sostenidamente, salvo los años 2016 y 2018, lo cual es positivo, pero la mejora es menos que proporcional al incremento de recursos que debieron usarse para este fin primordialmente. Además, el índice de mejora se mantiene por debajo del índice nacional, incluso de Lima Metropolitana, hasta el 2008. Los resultados de la gestión de servicios básicos en los locales escolares en Arequipa no son del todo positivos. La formación en ambientes sin servicios influye negativamente en los niños y jóvenes, al brindarles un concepto errado de aseo, cuidado personal y calidad de vida en general. El tener el dinero en caja parece confirmar la escasa capacidad de gestión del GRA en el sector educación.

En cuanto a calidad educativa, la región muestra índices de comprensión lectora y razonamiento matemático relativamente altos (superados solo por los de Moquegua y Tacna), con niveles del $46.7 \%$ y $43.1 \%$, respectivamente. En la figura 18 se muestra, sin embargo, que el conjunto del país y Lima Metropolitana han logrado mayores avances. 


\section{Figura 14}

Presupuesto de inversión de educación y ejecución presupuestal, 2005-2018 (millones de soles y \%)

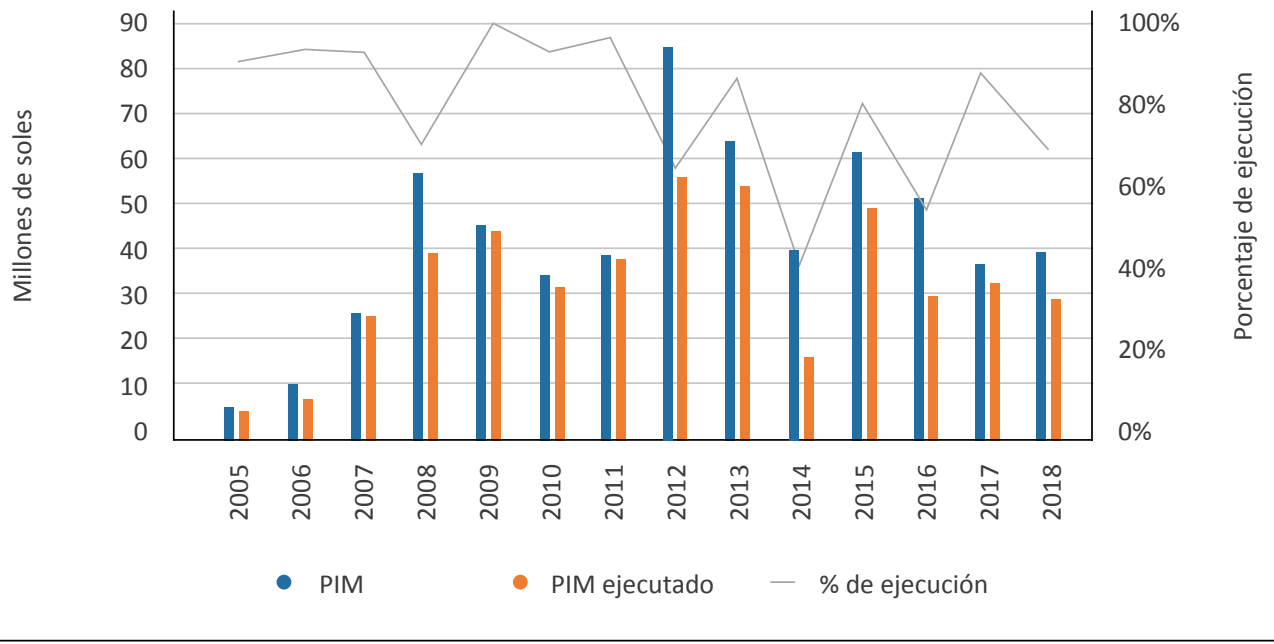

Nota. Elaboración propia basada en información del Ministerio de Economía y Finanzas (MEF).

\section{Figura 15}

Déficit de ejecución de inversiones en educación anual y acumulado GRA, 2005-2018 (millones de soles)

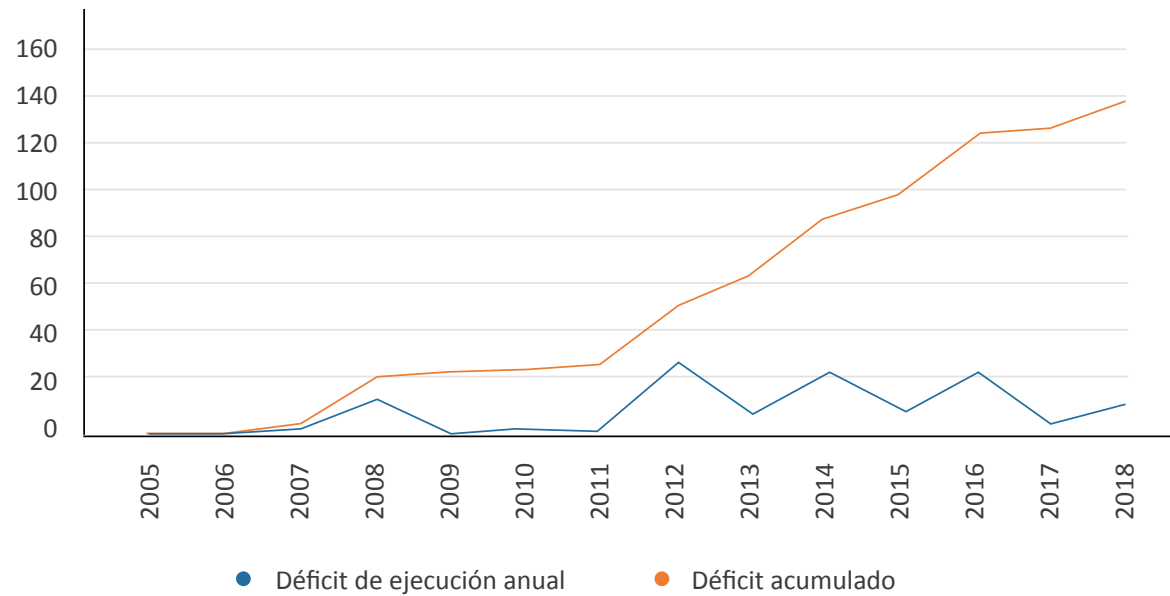

Nota. Elaboración propia basada en información del Ministerio de Economía y Finanzas (MEF). 


\section{Figura 16}

Presupuesto bienes y servicios, porcentaje de instituciones educativas (I.E.) en buen estado y con tres servicios básicos, 2005-2018

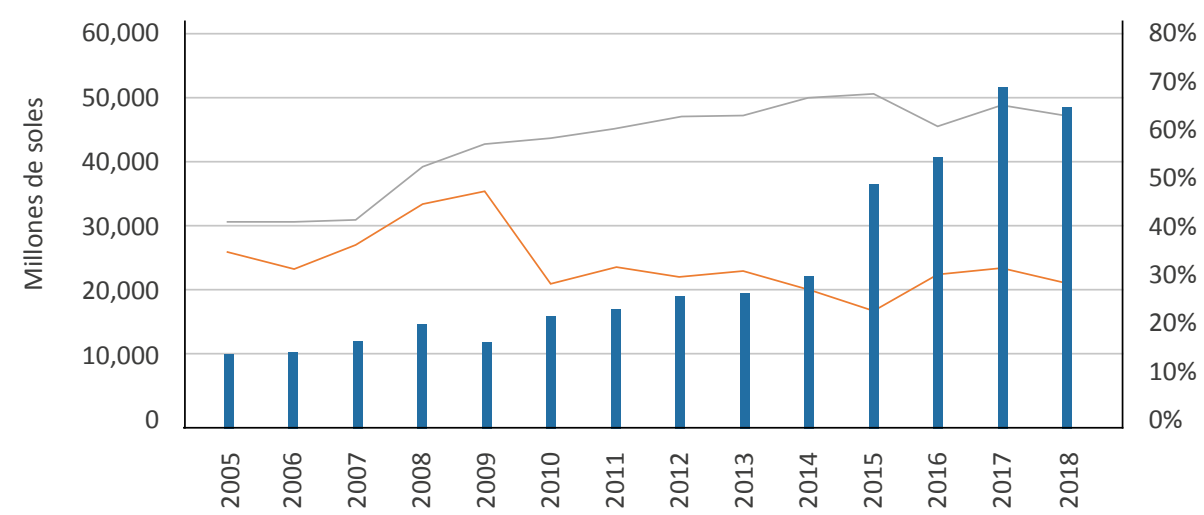

- Ppto. para bsyss $\quad-$ \% de I.E. en buen estado $\quad-\%$ de I.E. con tres servicios básicos

Nota. Elaboración propia basada en información del Ministerio de Economía y Finanzas (MEF) y del Ministerio de Educación, ESCALE, Unidad de Estadística Educativa.

\section{Figura 17}

Índice de mejora de locales escolares públicos con tres servicios básicos, 2005-2018 (2001=1)

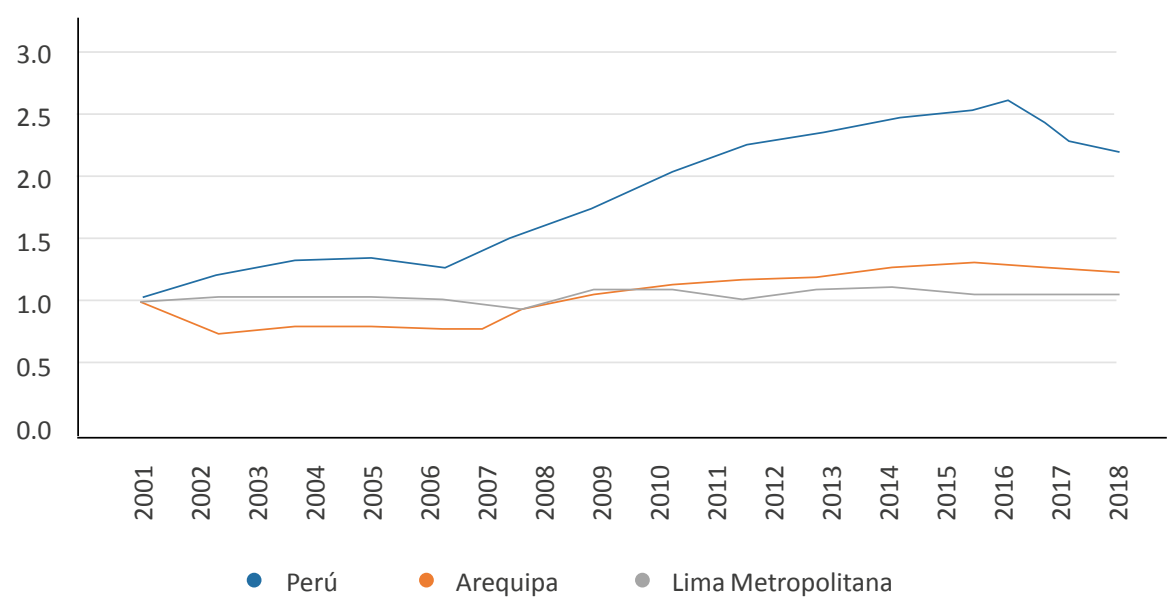

Nota. Elaboración propia basada en información del Ministerio de Educación, ESCALE, Unidad de Estadística Educativa. 


\section{Figura 18}

Índice de mejora de comprensión lectora comparativo: Perú, Lima Metropolitana y Arequipa, niños de segundo grado, 2007-2015 (2007=1)

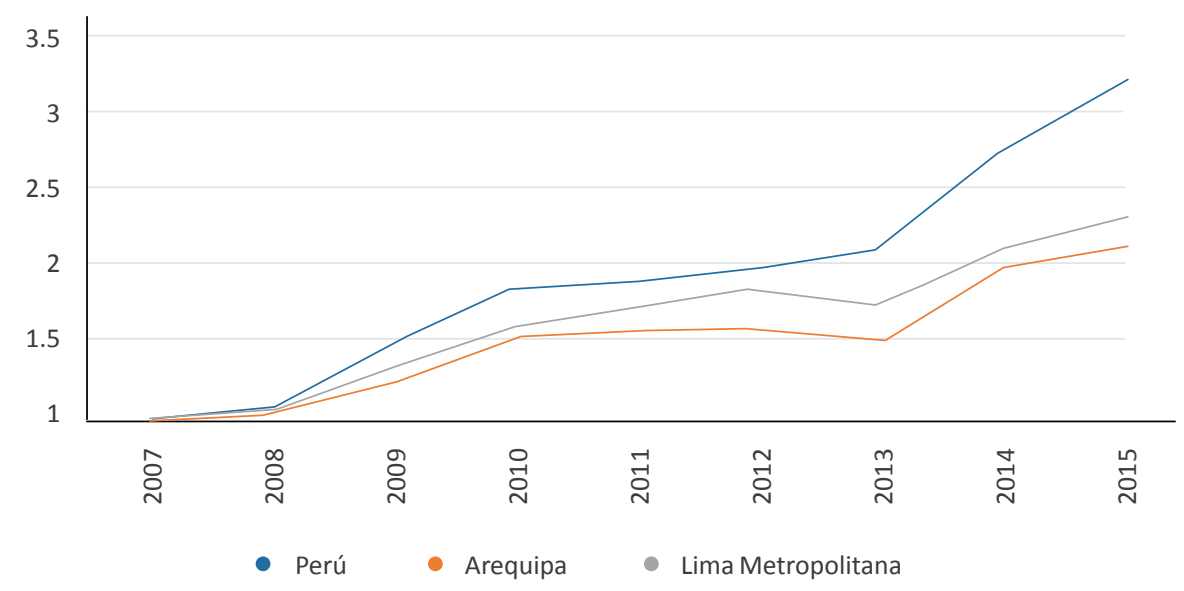

Nota. Elaboración propia basada en información del Ministerio de Educación, ESCALE, Unidad de estadística educativa.

Se puede concluir que el sector educación es el que más presupuesto ha recibido, pero no lo ha utilizado adecuadamente ni en cantidad (por las bajas ejecuciones presupuestales de inversiones) ni en calidad (por los magros resultados obtenidos, tanto en indicadores de infraestructura educativa, como en los cualitativos de comprensión lectora), pues se ha limitado a administrar conservadoramente lo que se recibió. Las razones deben ser materia de otras indagaciones, pero los resultados sugieren la falta de un planeamiento adecuado que vincule los diagnósticos con los objetivos, estrategias y acciones específicas, así como de un sistema de seguimiento que permita el conocimiento y control institucional y ciudadano.

En el año 2008 se aprobó el Proyecto Educativo Regional de Arequipa 2006-2021, que representó un nuevo esfuerzo; sin embargo, resultó estéril, al no recogerse sus mandatos en los planes estratégicos y operativos posteriores. No se han encontrado documentos que evidencien su seguimiento y monitoreo. Posteriormente, en el 2017 se retoma este esfuerzo con un horizonte hasta el año 2030, el mismo que, se espera, cumpla un rol más efectivo en el proceso educativo regional.

Puede considerarse, como otra consecuencia de esta gestión, el traslado importante de estudiantes de la región desde la educación pública hacia la educación privada. A pesar de los crecientes presupuestos, el alcance del servicio ha sido proporcionalmente menor. Se presenta la paradoja: a mayor presupuesto, menos estudiantes. Este cambio en las preferencias de las familias hacia la educación privada es un fenómeno repetido, sobre cuyas causas y consecuencias sería interesante investigar. (Véase figuras 19 y 20). 


\section{Figura 19}

Número de alumnos en educación pública en Arequipa, 2005-2018 (tabla en miles)

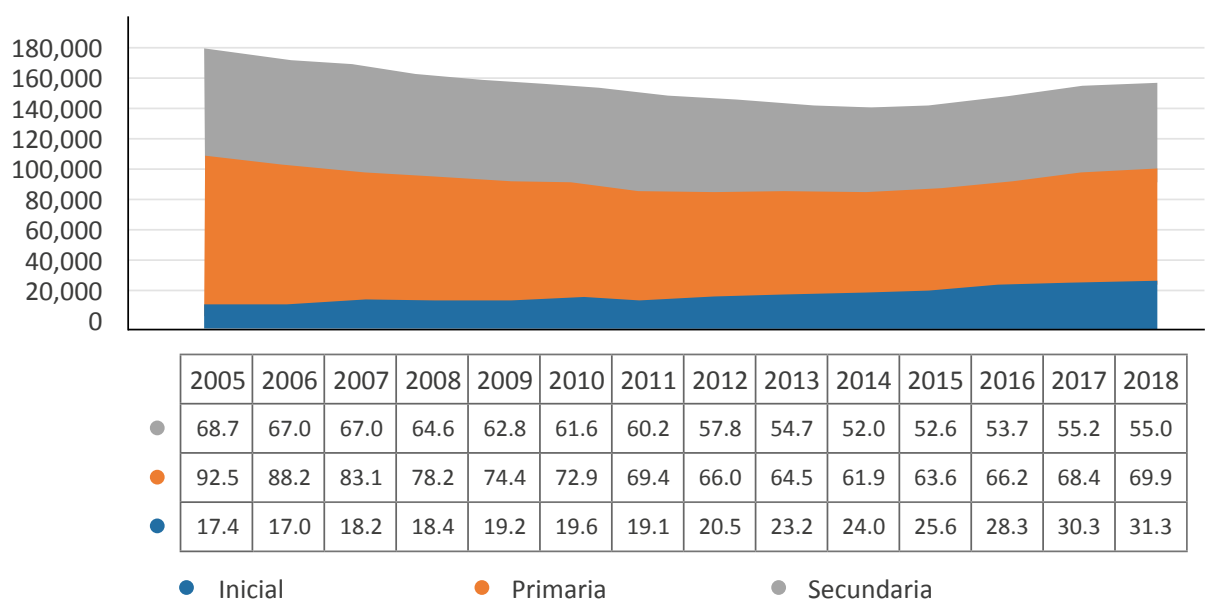

Nota. Elaboración propia basada en información del Ministerio de Educación, ESCALE, Unidad de Estadística Educativa.

\section{Figura 20}

Número de alumnos en educación privada en Arequipa, 2005-2018 (tabla en miles)

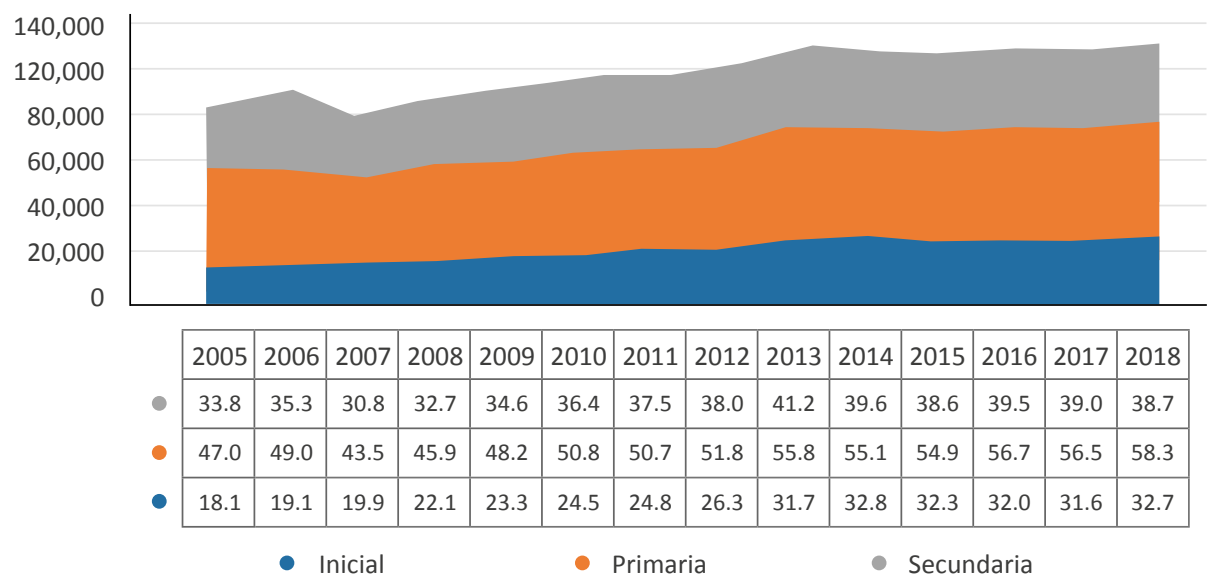

Nota. Elaboración propia basada en información del Ministerio de Educación, ESCALE, Unidad de estadística educativa. 


\subsection{El sector salud}

En salud, el diagnóstico del último plan regional señala que las principales causas de las carencias del sistema están relacionadas con el insuficiente personal especializado en las zonas alejadas, la falta de estrategia de atención y la limitada cantidad de establecimientos en las zonas rurales para atender a los más vulnerables, lo que generaría una elevada demanda no atendida.

Sin embargo, las estrategias y acciones no condicen con este diagnóstico; por el contrario, gran parte del presupuesto se concentra en los hospitales de la ciudad más grande de la región y en el instituto de enfermedades neoplásicas. El diagnóstico, las acciones estratégicas, y los proyectos y actividades aparecen desconectados, casi independientes. Una vez más, no se plantean indicadores para acciones operativas concretas que permitan el seguimiento y la participación ciudadana informada.

En las siguientes figuras se observa un presupuesto creciente a lo largo del período analizado y una ejecución fluctuante por encima del 90\%. Sin embargo, la falta de capacidad de ejecución de inversiones se repite, con un acumulado de 300 millones de soles no utilizados y los principales proyectos paralizados o no iniciados, con excepción del año 2006.

En cuanto al gasto corriente, en la figura 22 se compara la evolución del presupuesto para remuneraciones del sector con el incremento del

\section{Figura 21}

Evolución del presupuesto de salud y ejecución presupuestal, GRA, 2005-2018 (millones de soles y \%)

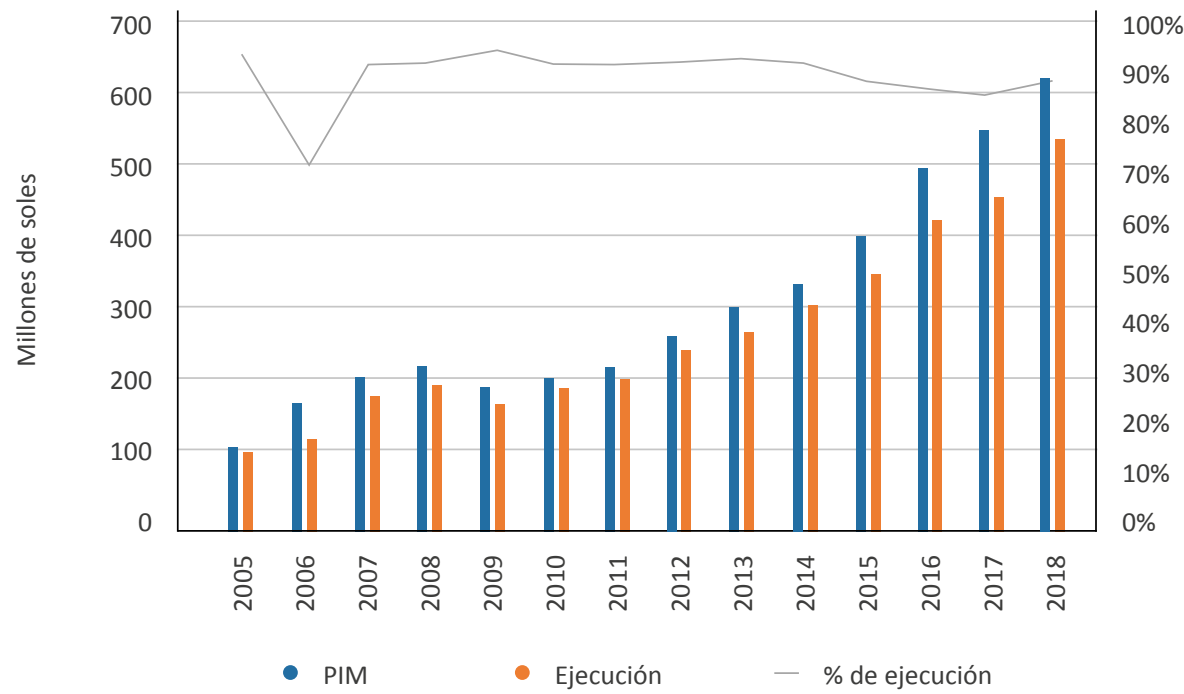

Nota. Elaboración propia basada en información del Ministerio de Economía y Finanzas (MEF). 


\section{Figura 22}

Presupuesto de remuneraciones y contratación del personal de salud, GRA, 2011-2018 (millones de soles)

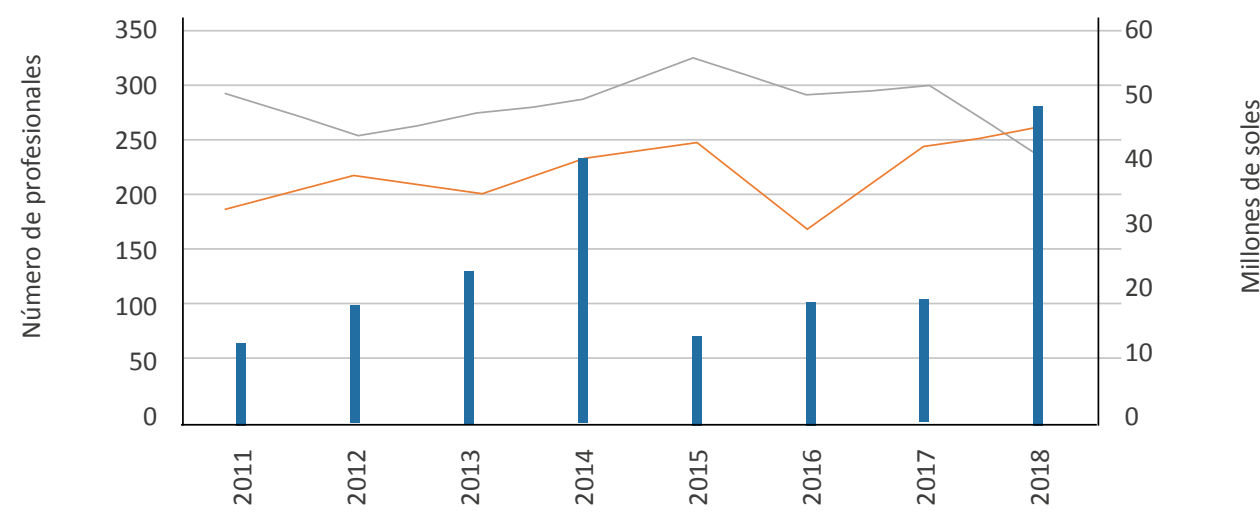

- Ppto. de remuneraciones - № de médicos contratados $\quad-\quad$ № de enfermeras contratadas

Nota. Elaboración propia basada en información del Ministerio de Economía y Finanzas (MEF) y del Instituto Nacional de Estadística e Informática (INEI), Sistema de Información para la Toma de Decisiones (SIRTOD).

número de médicos y enfermeras. En cálculos aproximados, se ha podido establecer el uso correcto de alrededor del $90 \%$ de los recursos que se destinaron efectivamente a la contratación de personal de salud. Lo que llama la atención es la poca relación entre los presupuestos anuales y la cantidad de personal contratado.

Es tarea fundamental del sector, el control de la salud de la infancia para evitar los problemas de desnutrición y anemia que azotan al Perú. En la figura 23 se ha relacionado el presupuesto para bienes y servicios (es decir, para compras y contrataciones de servicios específicos) con el número de atenciones para el control de la salud de la infancia. Se observa que, salvo los años 2017 y 2018, el monto presupuestado creció significativamente, pero no fue usado en este esfuerzo, ya que aparentemente no fue priorizado.
En este sector, la ejecución de inversiones ha sido también deficiente. Durante el período se dejó de utilizar recursos hasta acumular 300 millones de soles que debieron invertirse, como se muestra en la figura 24. El déficit de inversiones, la paralización de proyectos del sector y el escaso aumento de la oferta de camas hospitalarias sugieren la ineficacia en la utilización de los recursos públicos para la salud en la región.

Las consecuencias de las acciones tomadas por el GRA en el período se reflejan, en parte, en los indicadores mostrados en la tabla 9 , con resultados heterogéneos y volátiles.

El sistema de salud peruano se encuentra organizado según una norma técnica que considera niveles de atención en función a prevalencia y complejidad de las enfermedades, así como 


\section{Figura 23}

Tasas de crecimiento del presupuesto de gastos en bienes y del número de atenciones en el sector salud del GRA, 2008-2018

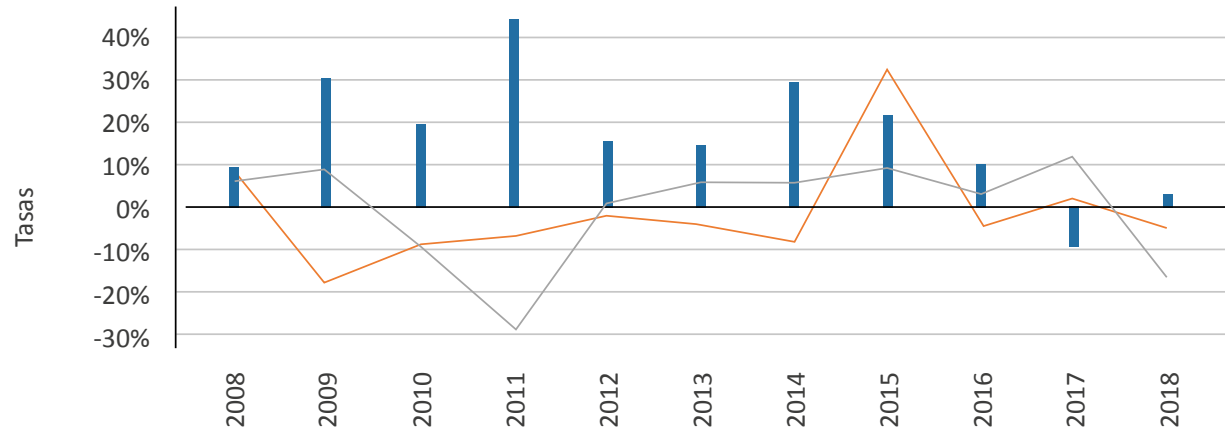

- Presupuesto de gastos en bienes y servicios

- Niños/as de 1 a 4 años que recibieron control de salud

- Niños/as menores de 1 año que recibieron control de salud

Nota. Elaboración propia basada en información del Ministerio de Economía y Finanzas (MEF) y del Instituto Nacional de Estadística e Informática (INEI), Sistema de Información para la Toma de Decisiones (SIRTOD).

\section{Figura 24}

Déficit de ejecución de inversiones en salud anual y acumulado GRA, 2005-2018 (millones de soles)

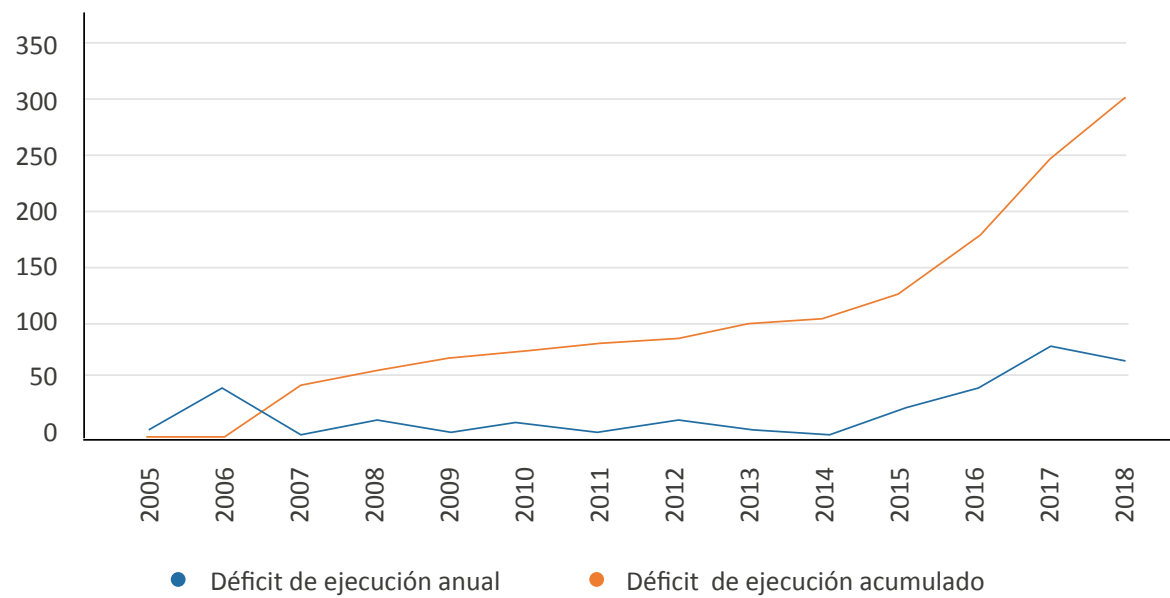

Nota. Elaboración propia basada en información del Ministerio de Economía y Finanzas (MEF). 


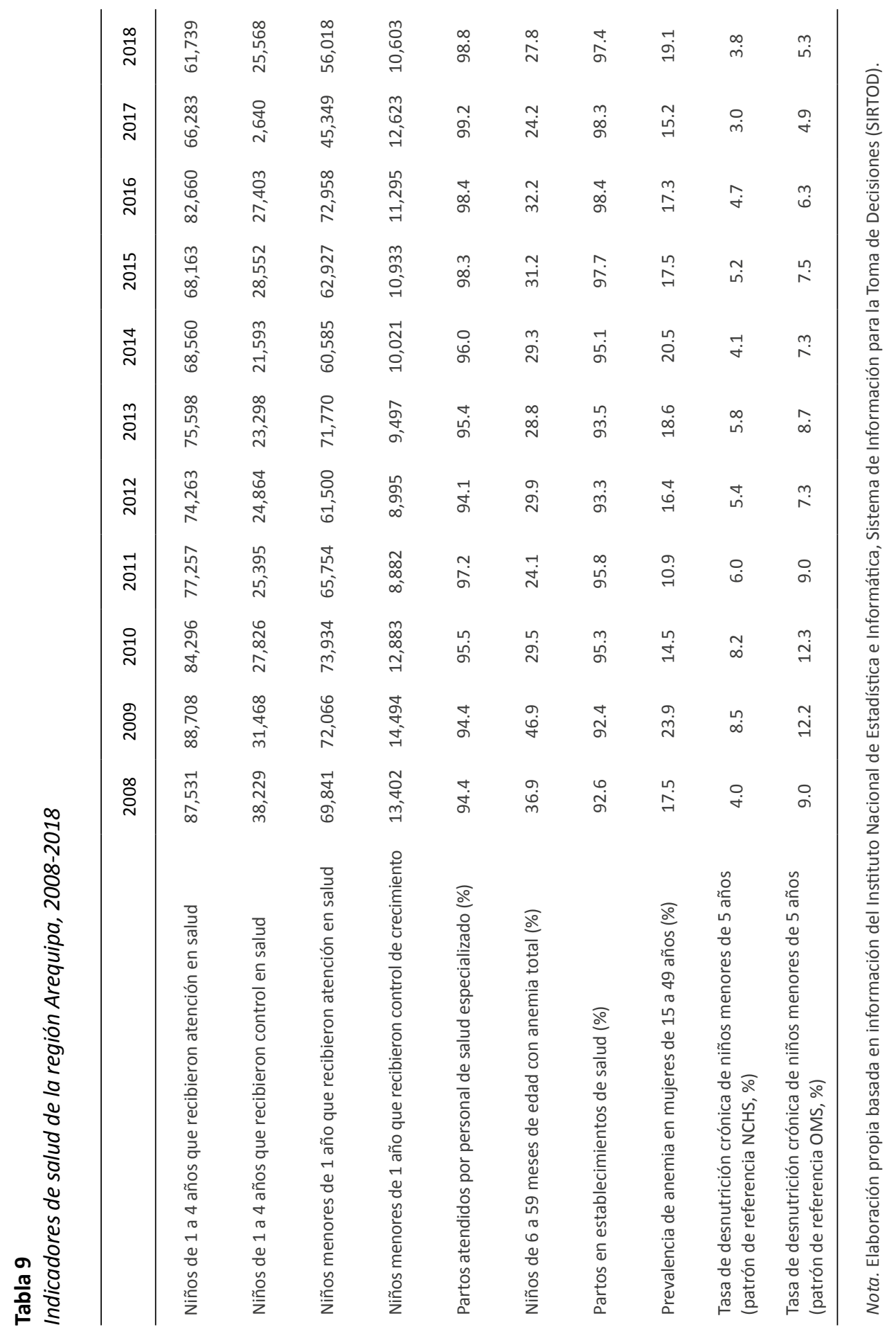




\section{Figura 25}

Número de establecimientos de salud del primer nivel de atención en Arequipa, 2005-2018

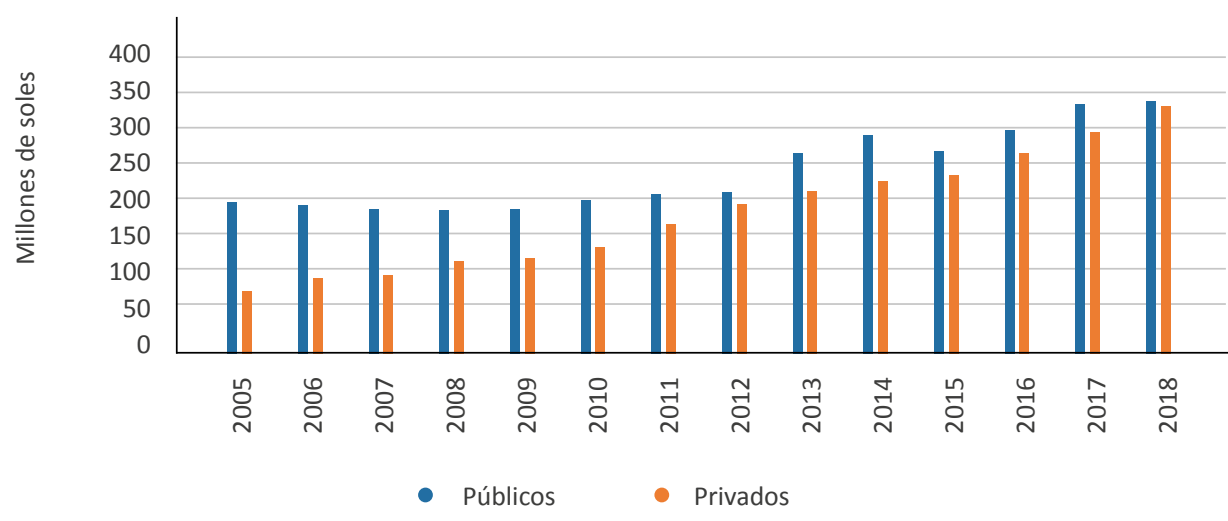

Nota. Se corrige el dato del 2016 por el promedio interanual ante una evidente falla de la fuente. Elaboración propia basada en información del Instituto Nacional de Estadística e Informática, Sistema de Información para la Toma de Decisiones (SIRTOD) y del Registro Nacional de Instituciones Prestadoras de Servicios de Salud (RENIPRESS).

a labores de detección precoz y promoción de la salud de la población. En esta línea, hay tres niveles de atención y ocho niveles de complejidad. El más importante, desde el punto de vista de cantidad de atenciones y resolución de problemas de salud, es el denominado primer nivel de atención, donde se atienden entre el $70 \%$ y el $80 \%$ de los casos de severidad y complejidad baja que no requieren especialistas ni equipamiento complejo (MINSA, 2004).

En la figura 25 se muestra que el número de establecimientos privados pasa de 71 en el 2003 a 332 en el 2018. Por otro lado, la oferta pública también crece, aunque en menor proporción, pasando de 197 a 336 establecimientos.

\section{Conclusiones}

El proceso de regionalización en Arequipa a través de la creación del gobierno regional no ha significado una mejora en la gestión de los recursos asignados para el desarrollo económico, por el contrario, ha ocasionado su desperdicio - al no ejecutar por completo los presupuestos asignados - y la paralización de los principales proyectos de desarrollo, tampoco ha propiciado un avance en la cobertura y calidad de los servicios básicos de educación y salud, evidenciándose un traslado de estudiantes y pacientes hacia el sector privado. El GRA siempre contó con proyectos viables vigentes y con financiamiento para ejecutarlos, sin embargo, su gestión no fue efectiva. 
El presupuesto público para la región Arequipa se ha incrementado constantemente y ha sido y sigue siendo administrado en partes casi iguales por el gobierno nacional y el regional. La porción administrada por el GRA, al cierre del período en estudio, se quintuplicó. Si se lo compara con lo asignado al conjunto de las otras regiones del país, Arequipa ha sido beneficiada presupuestalmente. Más relevante aún es el incremento continuo del presupuesto de inversiones, que pasó de solo 68 a 1,043 millones de soles, es decir, se multiplicó por quince.

Hasta el año 2011 la ejecución presupuestal regional se encontraba por encima de la nacional y de la del conjunto de gobiernos regionales; sin embargo, a partir del 2012 cae considerablemente hasta llegar al $80 \%$ en el 2018. En cuanto a ejecución de inversiones, el gobierno regional obtuvo su mejor ejecución en el año 2005 con $80 \%$ y la peor en el año 2018 con $58 \%$. Todos los años del período en análisis se ha dejado de usar recursos para inversión por un monto acumulado superior a los 2.2 mil millones de soles, que debieron haberse convertido en mejoras en los servicios públicos básicos y en la capacidad productiva regional.

La responsabilidad del GRA se concentra en cuatro sectores. Educación y salud en un primer nivel con el 52\% del presupuesto; en un segundo nivel de importancia, el sector transportes con $15 \%$ y el agropecuario con $10 \%$. Este último con una alta concentración en el proyecto Majes Siguas II. Sin embargo, la planeación existente considera prácticamente a todos los sectores, inclusive aquellos con presupuestos exiguos que generan interferencias con otras instancias de gobierno y con el sector privado.
El GRA contó, en el periodo 2005-2018, con 1,876 proyectos viables valorizados en 8,331 millones de soles, de los cuales se ejecutaron solamente 4,439 millones, es decir, el 53.3\%. En inversión, han predominado proyectos de transportes e infraestructura agrícola frente a los de educación y salud, que podrían manejarse con mayor efectividad por las autoridades regionales al encontrarse más cercanas a la población. Estos sectores fueron relegados por obras de infraestructura, necesarias pero que, debido a su complejidad, podrían haberse manejado más eficientemente desde el gobierno central.

En cuanto a la situación de los proyectos en función a lo que las normas públicas denominan «estado de la inversión», se ve que la mayor parte permaneció en condición de «activos» -que podrían haber constituido inversión-, de los cuales se ejecutó solamente el 54.6\%. De los proyectos considerados «cerrados», la mayoría no llegó a otorgar el bien público esperado. Del total de proyectos cerrados sin uso de recursos, el 70\% corresponde a educación. Existe también una importante proporción de los denominados «proyectos desactivados», que representa una pérdida por lo invertido en su diseño y tramitación.

Los principales proyectos de desarrollo regional se encuentran paralizados. Se podría afirmar que ningún proyecto de impacto económico o social ejecutado por el GRA se ha culminado, y los que están en operación lo hacen presionados por la necesidad.

La gestión de los sectores sociales ha sido deficiente al no tener planes estratégicos y operativos estructurados y coherentes. El GRA no ha elaborado diagnósticos adecuados que avancen hacia soluciones. Los montos de inver- 
sión presupuestados parecen insuficientes para el reto y su importancia para el desarrollo a largo plazo, aunque, paradójicamente, no se han utilizado en su integridad.

En educación, los presupuestos anuales fueron crecientes, pero no han tenido resultados satisfactorios en la misma proporción. Por el contrario, se dejó de invertir 136 millones de soles. Los avances en calidad educativa e infraestructura son escasos. Los locales en buen estado casi no se han incrementado — manteniendo su bajísimo nivel—y las mejoras en comprensión lectora están por debajo del promedio de mejora a nivel nacional. Finalmente, ha ocurrido una migración importante hacia la educación privada, produciéndose un efecto perverso: a más presupuesto público, menos población beneficiada.

En salud, la inexistencia de herramientas de gestión se repite. Los planes estratégicos no concuerdan con el diagnóstico ni, menos, con las acciones. Los principales proyectos de infraestructura no se han ejecutado o se encuentran paralizados. El monto de inversiones no ejecutadas y presupuestadas asciende a más de 300 millones de soles. Así pues, no hay una mejora homogénea de los indicadores de calidad en la proporción de lo esperado por el incremento presupuestal. Al igual que en educación, el sector privado ha pasado a ser un importante proveedor de servicios de atención primaria en salud debido, en parte, a las carencias del sistema público.

La gestión de esta instancia de gobierno debería concentrarse - mejorando las actuales competencias - en educación y salud y no en la ejecución de proyectos de gran envergadura — para lo cual, claramente, no reúne capacidades - ni en sectores para los que no cuenta con suficiente conocimiento ni recursos financieros, generando ineficiencias al interferir con otras instancias de gobierno, con empresas públicas y privadas e instituciones que actúan en su ámbito de influencia, pero sobre las que no tiene autoridad ejecutiva. Urge darle un norte claro a la gestión de los gobiernos regionales, los que, por encontrarse cercanos a la población, podrían cumplir un papel importante sobre todo en el desarrollo de los sectores sociales.

\section{Referencias bibliográficas y fuentes de consulta}

Apoyo consultoría (2017). Análisis del impacto de Sociedad Minera Cerro Verde en la economía de Arequipa y Perú (2005-2017).

Armijo, Daniela (2011). La gestión pública por resultados. ¿Cómo medir los resultados de la gestión gubernamental? Comisión Económica para América Latina, CEPAL.

Banco Central de Reserva del Perú [BCRP] (2003-2018). Series estadística. Lima. 
Centro Nacional de Planeamiento Estratégico [Ceplan] (2019). Perú 2030: Síntesis de tendencias globales y regionales. Lima: Congreso de la República.

Centro Nacional de Planeamiento Estratégico [Ceplan] (2013). Informe de evaluación del proceso de descentralización a 10 años de su inicio. Comisión de Descentralización, Regionalización, Gobiernos Locales y Modernización de la Gestión del Estado. Lima.

Gobierno Regional de Arequipa [GRA] (2003). Plan estratégico de desarrollo regional concertado 2003-2011. Arequipa.

Gobierno Regional de Arequipa [GRA] (2007-2017). Memorias de gestión. Arequipa.

Gobierno Regional de Arequipa [GRA] (2015). Plan de desarrollo regional concertado actualizado para la región Arequipa 2013-2021. Arequipa.

Contraloría General de la República [CGR] (2012). Estudio del proceso de descentralización en el Perú. Lima.

Instituto de Estadística e Informática [INEI] (varios años). SIRTOD. Sistema de Información Regional para la Toma de Decisiones.

Instituto de Estadística e Informática [INEI] (2005-2018). Encuesta Nacional de Hogares. Lima.

Instituto de Estadística e Informática [INEI] (2018). Censo Nacional de Población y Vivienda. Lima.

Instituto de Estadística e Informática [INEI] (2021). Evolución de la Pobreza Monetaria 2009-2020. Informe técnico.

Ministerio de Economía y Finanzas [MEF] (2005-2018). Búsqueda amigable. Ejecución presupuestal. Consulta de inversiones. Lima.

Ministerio de Economía y Finanzas [MEF]. Preguntas frecuentes. SNIP y gobiernos locales. Dirección Nacional de Presupuesto Público. Lima.

Ministerio de Economía y Finanzas [MEF] (2015). Compendio de normatividad del Sistema Nacional de Inversión Pública. Viceministerio de Economía. Dirección Nacional de Inversión Pública. Lima.

Ministerio de Educación [MINEDU] (varios años). Escale. Unidad de Estadística Educativa. Lima.

Ministerio de Salud [MINSA] (2004). Categorías de establecimientos del Sector Salud. N T N. 0021 - MINSA / DGSP V.01. Lima: Dirección General de Salud de las Personas.

Ministerio de Salud [MINSA] (varios años). Registro Nacional de Instituciones Prestadoras de Servicios de Salud. RENIPRESS. Lima.

Molina Martínez, Raúl (2016). Análisis comparativo de balances sobre el proceso de descentralización. Lima. 
Presidencia del Consejo de Ministros (2018). Informe anual de proceso de descentralización 2017. Secretaría de Descentralización. Lima.

Normas y Leyes

Ley N. ${ }^{\circ}$ 27783. Ley de Bases de la Descentralización.

Ley N.²7867. Ley Orgánica de Gobiernos Regionales.

Ley N.²6922. Ley Marco de Descentralización.

Ministerio de Economía y Finanzas. Instructivos N. ${ }^{\circ}$ 001-2019-EF/63.01 y N. ${ }^{\circ} 002-2017-E F / 63.01$. Aplicaciones Informáticas del Banco de Inversiones, Dirección General de Inversión Pública.

Ministerio de Salud. Categorías de establecimientos del Sector Salud. N T N. 0021- MINSA / DGSP V.01. Lima: Dirección General de Salud de las Personas, MINSA, 2004. 